\title{
Networked Control under Random and Malicious Packet Losses
}

\author{
Ahmet Cetinkaya, Hideaki Ishii, and Tomohisa Hayakawa
}

\begin{abstract}
We study cyber security issues in networked control of a linear dynamical system. Specifically, the dynamical system and the controller are assumed to be connected through a communication channel that face malicious attacks as well as random packet losses due to unreliability of transmissions. We provide a probabilistic characterization for the link failures which allows us to study combined effects of malicious and random packet losses. We first investigate almost sure stabilization under an event-triggered control law, where we utilize Lyapunov-like functions to characterize the triggering times at which the plant and the controller attempt to exchange state and control data over the network. We then provide a look at the networked control problem from the attacker's perspective and explore malicious attacks that cause instability. Finally, we demonstrate the efficacy of our results with numerical examples.
\end{abstract}

\section{INTRODUCTION}

Cyber security has become a critical problem in industrial processes, since nowadays they incorporate information and communication technologies that are prone to cyber threats. Cyber attacks can disrupt the normal operation of services that are critical to the society as they can cause financial losses and environmental damages. It is thus essential to ensure cyber security of existing infrastructures and design new cyberattack-resilient ones.

Literature on cyber security points out cyber threats against industrial control systems utilized in many fields (see [1] and the references therein). Vulnerabilities of the channels used for transmission of measurement and control data pose a critical issue for the security of control systems. This is because the channels are recently connected via the Internet or wireless communications [2], [3]. Communication channels, for instance, may face jamming attacks initiated by malicious agents [4], [5]. Such attacks block the communication link and effectively prevent transmission of packets between the plant and the controller. It is mentioned in [5] that jamming attacks pose a major security threat, as they can be easily performed with devices that target various wireless communication protocols. In recent works [6]-[13], networked control problems under jamming attacks were investigated using control and/or game-theoretic methods. However, jamming may not be the only cause of malicious packet losses. Compromised routers in a network may also intentionally drop packets [14], [15]. The work [16] explored the control problem over a multihop

A. Cetinkaya and T. Hayakawa are with the Department of Mechanical and Environmental Informatics, Tokyo Institute of Technology, Tokyo 152-8552, Japan. ahmet@dsl.mei.titech.ac.jp, hayakawa@mei.titech.ac.jp

$\mathrm{H}$. Ishii is with the Department of Computational Intelligence and Systems Science, Tokyo Insitute of Technology, Yokohama, 226-8502, Japan. ishii@dis.titech.ac.jp

This work was supported in part by Japan Science and Technology Agency under the EMS-CREST program. network with malicious nodes that intentionally stop forwarding packets or alter packet contents.

In addition to actions of malicious agents, state measurement and control input packets may also fail to be transmitted at times due to network congestion or errors in communication. Stochastic models provide accurate characterization of such nonmalicious network issues [17], [18]. In the literature, unreliability of a network is often characterized through random models for packet loss events [19], [20]. For instance, in [21][23], Bernoulli processes are used for modeling packet losses in a network. Furthermore, in [24], [25], packet loss events are characterized in a more general way by employing Markov chains. In those studies, a variety of control methods are also proposed to ensure stability of networked control systems that face random packet losses.

In this paper, we propose a stochastic representation of packet transmission failures in a network between a plant and a controller. Our proposed model is sufficiently general and allows us to explore some of the existing random and malicious packet loss scenarios in a unified manner. At the core of this characterization, we have a tail probability condition on the average number of state measurement and control input packet failures in the network. We demonstrate that random packet losses, malicious attacks, as well as the combination of those two phenomena satisfy the condition with different parameters. We model random losses by using a binary-valued timeinhomogeneous Markov chain. Furthermore, to characterize malicious attacks, we use a model similar to the one in [10]. Specifically, this model allows attacks to happen arbitrarily as long as the total number of packet exchange attempts that face malicious attacks are almost surely bounded by a certain ratio of the number of total packet exchange attempts between the plant and the controller. The almost sure bound used in our model in fact allows not only deterministic strategies but also stochasticity in the generation of malicious attacks. As a result, the model captures attacks that are generated based on randomly varying information such as state and control input or the random packet losses. Besides, an attacker may also intentionally use randomness to imitate packet losses that occur due to congestion or channel noise.

Through our malicious attack model, we consider scenarios where the attacker targets the network only when the plant and the controller attempt to exchange packets. In a jamming attack scenario, our characterization, hence, can be considered as a model for reactive jamming discussed in [4] for wireless networks. The classification in [4] divides attackers into two groups: active and reactive ones. An active jamming attacker tries to block a communication channel regardless of whether the channel is being used or not, whereas a reactive attacker continuously monitors the channel and attacks only when there is transmission. It is mentioned in [4] that it may be harder 
to detect a reactive jamming attacker as packets may also be lost due to nonmalicious network issues and hence the reason for packet losses may not be known with certainty. A similar issue where packet losses occur due to both malicious and nonmalicious reasons exists also in the context of multihop networks. For instance [15] investigates combined effects of malicious packet drops and nonmalicious channel errors.

Motivated by the scenarios mentioned above, we utilize our probabilistic characterization also to investigate networks that are subject to the combination of random transmission errors due to unreliability of the channel and attacks conducted by malicious agents. In our analysis, we consider two cases: (i) when the attacks and random packet losses are modeled as independent processes and (ii) when the attack strategy is dependent on the random packet losses. The dependent case is essential to model the situation where the attacker has information of the random packet losses in the communication channel and utilizes this information in the attack strategy. Furthermore, we may also consider situations when the attacker decides to attack based on the content of packets. In the case of jamming attacks, this corresponds to selective jamming discussed in [26], [27], where the intelligent jamming attacker listens to the communication channel and decides whether to interfere or not depending on the packet being transmitted. For example, a jamming attacker may decide not to interfere with the communication when the packet being transmitted is already corrupted by channel noise. Moreover, in a network of multiple nodes malicious ones may intentionally drop certain packets based on their content [28]. The main theoretical challenge in dealing with the combination of random packet losses and malicious attacks stems from the fact that these two phenomena are of different nature and hence have different models. By utilizing a tail probability inequality for the sum of processes that represent random packet losses and malicious attacks, we show that our proposed probabilistic characterization allows us to deal with both independent and dependent loss cases.

By utilizing our probabilistic packet transmission model, we investigate the networked control problem of a linear plant through an event-triggered framework. Event-triggered control methods have recently been employed in many studies (see [29]-[31] and the references therein). We follow the approach in [32], [33] and utilize Lyapunov-like functions to determine the triggering times at which the plant and the controller attempt to exchange state and control input information. The triggering conditions that we propose ensure that the value of a Lyapunov-like function of the state stays within certain limits. Packet exchanges are attempted only before the value of the Lyapunov-like function is predicted to exceed the limit. In a successful packet exchange scenario, state measurements are sent from the plant to the controller, which computes a control input and sends it back to the plant. However, state measurement or control input packets may fail to be transmitted due to random packet losses and malicious attacks.

Our packet failure characterization and control system analysis differ from those of the recent studies [34]-[36], which also investigate the event-triggered control problem under packet losses. Specifically, in [34], the number of consecutive packet losses is assumed to be upper-bounded, and a deterministic Lyapunov function approach is used for the closed-loop stability analysis. Moreover, in [35], [36] the packet losses are modeled by a Bernoulli process. The stability analysis in [36] is based on investigating the evolution of the expectation of a Lyapunov function. Despite the similarity to our malicious attack model, our stability analysis also differs from that of [10], where the analysis relies on a deterministic approach for obtaining an exponentially decreasing upper bound for the norm of the state. Our approach for stability analysis is related to obtaining an upper bound on the top Lyapunov exponent (see [37]-[39]) of the system and in that sense it is more similar to the stability analysis conducted in [23], [40] for networked systems without event-triggering. Specifically, we find a stochastic upper bound for a Lyapunov-like function and show that this stochastic upper bound tends to zero under certain conditions indicating almost sure asymptotic stability.

In addition to stability analysis, we also address the question of finding instability conditions under which the state of the closed-loop system diverges almost surely. We observe that an attack strategy that causes sufficiently frequent packet losses can destabilize the closed-loop dynamics. This instability result allows us to investigate effects of potential malicious attacks on a networked control system.

The rest of the paper is organized as follows. In Section II we describe the networked control problem under random and malicious packet losses. We present an event-triggered control framework and provide sufficient conditions for almost sure asymptotic stability of the closed-loop system in Section III. In Section IV, we look at the networked problem from the attacker's perspective and provide conditions for instability of the system. We present illustrative numerical examples in Section V. Finally, in Section VI, we conclude the paper.

We note that part of the results in Sections [II and [II] appeared without proofs in our preliminary report [41]. Here, we provide a more detailed discussion with complete proofs.

We use a fairly standard notation in the paper. Specifically, we denote positive and nonnegative integers by $\mathbb{N}$ and $\mathbb{N}_{0}$, respectively. Moreover, $\|\cdot\|$ denotes the Euclidean vector norm and $\lfloor\cdot\rfloor$ denotes the largest integer that is less than or equal to its real argument. The notation $\mathbb{P}[\cdot]$ denotes the probability on a probability space $(\Omega, \mathcal{F}, \mathbb{P})$ with filtration $\left\{\mathcal{F}_{i}\right\}_{i \in \mathbb{N}_{0}}$ such that $\mathcal{F}_{i_{1}} \subset \mathcal{F}_{i_{2}} \subset \mathcal{F}$ for $i_{1}, i_{2} \in \mathbb{N}_{0}$ with $i_{1}<i_{2}$.

\section{Networked Control Problem And CHARACTERIZATION OF NETWORK WITH RANDOM AND Malicious PaCKET Losses}

In this section we introduce the networked control problem and present a characterization for a network with random packet losses and those caused by malicious agents.

\section{A. Networked Control System}

Consider the linear dynamical system

$$
x(t+1)=A x(t)+B u(t), \quad x(0)=x_{0}, \quad t \in \mathbb{N}_{0},
$$

where $x(t) \in \mathbb{R}^{n}$ and $u(t) \in \mathbb{R}^{m}$ denote the state and the control input, respectively; furthermore, $A \in \mathbb{R}^{n \times n}$ and $B^{n \times m}$ are the state and input matrices, respectively. 
In our networked control problem, the plant and the controller exchange information packets over a communication channel to achieve stabilization of the zero solution $x(t) \equiv 0$. We consider the case where packets are transmitted without delay, but they may get lost. In a successful packet exchange scenario, at a certain time instant, measured plant states are transmitted to the controller, which generates a control input signal and sends it to the plant. The transmitted control input is applied at the plant side. In the case of an unsuccessful packet exchange attempt, either the measured state packet or the control input packet may get dropped, and in such cases control input at the plant side is set to 0 , which is a common approach in the literature (e.g., [20], [24], [25], [40]). In this setup, the plant is informed about a packet exchange failure by the lack of an incoming control input. Specific acknowledgement messages are thus not needed. This allows the practical implementation by using a UDP-like communication protocol discussed in [19].

We use $\tau_{i} \in \mathbb{N}_{0}, i \in \mathbb{N}_{0}$, (with $\tau_{i}<\tau_{i+1}$ ) to denote the time instants at which packet exchanges between the plant and the controller are attempted. In this paper, we consider both the case where packet exchanges are attempted at all time instants and the case where an event-triggering mechanism decides the successive packet exchange attempt times. In both cases, the control input $u(t)$ applied to the plant is given by

$$
u(t) \triangleq(1-l(i)) K x\left(\tau_{i}\right), t \in\left\{\tau_{i}, \ldots, \tau_{i+1}-1\right\},
$$

where $K \in \mathbb{R}^{m \times n}$ denotes the feedback gain and $\{l(i) \in$ $\{0,1\}\}_{i \in \mathbb{N}_{0}}$ is a binary-valued process that characterizes success or failure of packet exchange attempts. When $l(i)=0$, the packet exchange attempt at time $\tau_{i}$ is successful and the piecewise-constant control input at the plant side is set to $u\left(\tau_{i}\right)=K x\left(\tau_{i}\right)$. On the other hand, $l(i)=1$ indicates that either the packet sent from the plant or the packet sent from the controller is lost at time $\tau_{i}$. Again, in such situations, control input at the plant side is set to 0 . We emphasize that the framework described above allows us to deal with dropouts in both state and control input channels of the network illustrated in Fig. 1. In particular, the process $l(\cdot)$ is an overall indicator of the packet exchange failures over these channels.

\section{B. Network Characterization}

Packet transmission failures in a network may have different reasons. In what follows we characterize the effects of certain stochastic and malicious packet loss models in a unified manner by exploring dynamical evolution of the total number of packet exchange failures.

First, we define a nonnegative integer-valued process $\left\{L(k) \in \mathbb{N}_{0}\right\}_{k \in \mathbb{N}}$ by

$$
L(k) \triangleq \sum_{i=0}^{k-1} l(i), \quad k \in \mathbb{N} .
$$

Note that $L(k)$ denotes the total number of failed packet exchange attempts during the time interval $\left[0, \tau_{k-1}\right]$, where $k$ attempts have been made.

In our packet loss model, we place a bound on the ratio of failed attempts in a probabilistic and asymptotic sense.
Assumption 2.1: There exists a scalar $\rho \in[0,1]$ such that

$$
\sum_{k=1}^{\infty} \mathbb{P}[L(k)>\rho k]<\infty .
$$

The condition (4) provides a probabilistic characterization of the evolution of the total number of packet exchange failures through the scalar $\rho \in[0,1]$, representing their average ratio. Note also that (4) describes a condition on the tail probability $\mathbb{P}[L(k)>\rho k]=\mathbb{P}\left[\frac{L(k)}{k}>\rho\right]$ of loss ratio $\frac{L(k)}{k}$. This condition is sufficiently general and includes some of the existing packet loss models in the literature. We illustrate its generality by establishing that condition (4) holds for four different cases:

1) random packet losses,

2) malicious packet losses,

3) combination of the two losses in 1) and 2) when they are independent, and finally

4) combination but when they are dependent.

Note that for any packet loss model, Assumption 2.1 is trivially satisfied with $\rho=1$, since $\mathbb{P}[L(k)>k]=0$. On the other hand, as we see below, for certain random and malicious packet loss models, $\rho$ can be obtained to be strictly smaller than 1 . A closely related characterization for packet dropouts is presented in [23]; the scalar $\rho$ in (4) corresponds to the notion of dropout rate discussed there.

1) Random Packet Losses: To characterize nonmalicious network issues such as packet drops due to network congestion or communication errors, we utilize time-inhomogeneous Markov chains. Specifically, let $\left\{l_{\mathrm{R}}(i) \in\{0,1\}\right\}_{i \in \mathbb{N}_{0}}$ be a time-inhomogeneous Markov chain adapted to filtration $\left\{\mathcal{F}_{i}\right\}_{i \in \mathbb{N}_{0}}$. Here, the $\sigma$-algebra $\mathcal{F}_{i}$ contains all random packet transmission success/failure events for the first $i+1$ packet exchange attempt times $\left\{\tau_{0}, \tau_{1}, \ldots, \tau_{i}\right\}$. The Markov chain $\left\{l_{\mathrm{R}}(i) \in\{0,1\}\right\}_{i \in \mathbb{N}_{0}}$ is characterized by initial distributions $\vartheta_{q} \in[0,1], q \in\{0,1\}$, and time-varying transition probabilities $p_{q, r}: \mathbb{N}_{0} \rightarrow[0,1], q, r \in\{0,1\}$, such that

$$
\begin{aligned}
\mathbb{P}\left[l_{\mathrm{R}}(0)\right. & =q]=\vartheta_{q}, \\
\mathbb{P}\left[l_{\mathrm{R}}(i+1)=r \mid l_{\mathrm{R}}(i)\right. & =q]=p_{q, r}(i), \quad i \in \mathbb{N}_{0} .
\end{aligned}
$$

The state $l_{\mathrm{R}}(i)=1$ indicates that the network faces random packet losses at time $\tau_{i}$, and hence the packet exchange attempt at $\tau_{i}$ results in failure. Here, success/failure of a packet exchange attempt depends on the states of the previous packet exchange attempts. Furthermore, transition probabilities between success $\left(l_{\mathrm{R}}(i)=0\right)$ and failure $\left(l_{\mathrm{R}}(i)=1\right)$ states are timedependent. It is important to note that the time-inhomogeneous Markov chain characterization with time-varying transition probabilities allows us to take into account the variation in the network between consecutive packet transmission instants. Furthermore, this characterization generalizes the Bernoulli and time-homogeneous Markov chain models that are often used in the literature.

In what follows we show that Assumption 2.1 is satisfied when the network faces random packet losses described by time-inhomogeneous Markov chains. In characterization of the scalar $\rho$ used in Assumption 2.1 we use upper-bounds 
for transmission failure and success probabilities denoted respectively by $p_{1} \in[0,1]$ and $p_{0} \in[0,1]$ such that

$$
\begin{aligned}
& p_{q, 1}(i) \leq p_{1}, \\
& p_{q, 0}(i) \leq p_{0}, \quad q \in\{0,1\}, \quad i \in \mathbb{N}_{0} .
\end{aligned}
$$

Note that even though $p_{q, r}(i)$ provide precise information about the transitions between the states of random packet losses, this information cannot be utilized when the network faces the combination of malicious attacks and random packet losses (discussed in Sections II-B3 and II-B4). In such cases, information about the probability of malicious attacks for each transmission attempt is not available, and as a result, transition probabilities $p_{q, r}(i)$ for random packet losses cannot be utilized to obtain the overall packet exchange failure probabilities. On the other hand, we can employ the upperbounds $p_{1}$ and $p_{0}$ when we show that the overall packet exchange failures satisfy Assumption 2.1

Lemma 2.1: For the time-inhomogeneous process $\left\{l_{\mathrm{R}}(i) \in\right.$ $\{0,1\}\}_{i \in \mathbb{N}_{0}}$ with transmission failure probability upper-bound $p_{1} \in(0,1)$ that satisfy $(6)$, we have

$$
\sum_{k=1}^{\infty} \mathbb{P}\left[\sum_{i=0}^{k-1} l_{\mathrm{R}}(i)>\rho_{\mathrm{R}} k\right]<\infty,
$$

for all $\rho_{\mathrm{R}} \in\left(p_{1}, 1\right)$.

Proof: We use Lemma A.1 in the Appendix to prove this result. Specifically, let $\tilde{p}=p_{1}, \tilde{w}=1$, and define the processes $\{\xi(i) \in\{0,1\}\}_{i \in \mathbb{N}_{0}}$ and $\{\chi(i) \in\{0,1\}\}_{i \in \mathbb{N}_{0}}$ with $\xi(i)=l_{\mathrm{R}}(i)$ and $\chi(i)=1, i \in \mathbb{N}_{0}$. Since the conditions in (67) and (68) are satisfied, it follows from Lemma A.1 that $\mathbb{P}\left[\sum_{i=0}^{k-1} l_{\mathrm{R}}(i)>\rho_{\mathrm{R}} k\right] \leq \psi_{k}$, where $\psi_{k} \triangleq \phi^{-\rho_{\mathrm{R}} k+1} \frac{\left(\left(\phi_{1}-1\right) p_{1}+1\right)^{k}-1}{(\phi-1) p_{1}}$ with $\phi \triangleq \frac{\rho_{\mathrm{R}}\left(1-p_{1}\right)}{p_{1}\left(1-\rho_{\mathrm{R}}\right)}$, and $\sum_{k=1}^{\infty} \psi_{k}<\infty$, which implies (8).

Lemma 2.1 indicates that when packet exchange failures occur due to random packet losses (i.e., $l(i)=l_{\mathrm{R}}(i)$ ), Assumption 2.1 holds for all $\rho \in\left(p_{1}, 1\right)$.

2) Packet Losses Due to Malicious Activity: Packet transmissions in a channel may get interrupted due to malicious activities. For example, a compromised router in a network may deny to forward incoming packets. In addition, packet losses may also be caused by jamming attacks. A model for the attack strategy of a malicious agent has been proposed in [10]. In that study, the sum of the length of attack durations is assumed to be bounded by a certain ratio of total time.

By following the approach of [10], let $\left\{l_{\mathrm{M}}(i) \in\{0,1\}\right\}_{i \in \mathbb{N}_{0}}$ denote the state of attacks. The state $l_{\mathrm{M}}(i)=1$ indicates that the packet transmission faces an attack at time $\tau_{i}$. We consider the case where the number of packet exchange attempts that face attacks are upper bounded almost surely by a certain ratio of the total number of packet exchange attempts, that is, $\left\{l_{\mathrm{M}}(i) \in\{0,1\}\right\}_{i \in \mathbb{N}_{0}}$ satisfies

$$
\mathbb{P}\left[\sum_{i=0}^{k-1} l_{\mathrm{M}}(i) \leq \kappa+\frac{k}{\tau}\right]=1, \quad k \in \mathbb{N},
$$

where $\kappa \geq 0$ and $\tau>1$. In this characterization, among $k$ packet exchange attempts, at most $\kappa+\frac{k}{\tau}$ of them are affected by attacks. Note that when $\kappa=0$, 9 implies no attack in the beginning: $l_{\mathrm{M}}(i)=0, i \in\{0, \ldots,\lfloor\tau\rfloor\}$, almost surely. Scenarios that involve possible attacks during the first few packet exchange attempts can be modeled by setting $\kappa>0$.

In what follows, we would like to highlight the relations of the malicious packet loss model in (9) to those in the literature. First, since the attacks only happen at packet exchange attempt instants, the characterization in (9) can be considered as a reactive jamming model [4], where the attacker attacks the channel only when there is a packet being transmitted. To avoid being detected, an attacker may refrain from causing all packets to be lost. The ratio $\frac{1}{\tau}$ in (9) characterizes the average portion of the packet transmission attempts that face attacks. Furthermore, in the case of jamming attacks, in addition to avoid being detected, the attacker may also need to take into account the energy requirements of jamming. The ratio $\frac{1}{\tau}$ in this case corresponds to the notion jamming rate discussed in [42], and it is related to the energy usage of the jammer.

Remark 2.2: A packet loss model that may be used to capture behavior of an intelligent attacker is also discussed in [21], where transmissions between the plant and the controller are attempted at all time instants and the proposed model allows packet losses to occur arbitrarily as long as the lengths of intervals between consecutive successful packet transmissions are not more than a given fixed length. A similar model has also been used in [34], where an event-triggered control method is used and the number of consecutive packet losses is assumed to be upper-bounded by a constant. Note that the packet loss model discussed in [21], [34] can be described within the framework provided by (9) through setting $\tau=\frac{s+1}{s}$, where $s \geq 1$ denotes the upper-bound on the number of consecutive packet losses. Under this setting, the condition (9) provides more freedom to the attacker as it does not necessarily require lengths of intervals between consecutive successful packet transmission times to be upperbounded by a fixed constant. In fact for any $\tau>1$, (9) allows the attacker to cause any number of consecutive packet losses after waiting sufficiently long without attacking. Notice that the number of consecutive packet losses is not restricted to be bounded also in the case of random packet loss models (see Section II-B1, as well as [20], [24], [43]).

As pointed out in [10], the condition (9) also shares some similarities with the socalled average dwell time condition [44] utilized in switched systems. In switched systems, the average dwell time condition requires the number $N\left(k_{2}, k_{1}\right)$ of switches in between times $k_{1}$ and $k_{2} \geq k_{1}$ to satisfy

$$
N\left(k_{2}, k_{1}\right) \leq \kappa+\frac{k_{2}-k_{1}}{\tau}, \quad k_{2} \geq k_{1} \geq 0,
$$

where $\tau>0$ denotes the average dwell time. The inequality (10) guarantees that the switches occur slowly on average. In this study, we do not require a condition on the number of switches between packet exchange success and failure states. Rather than that we utilize (9), which is a condition on the total number of packet exchange failures due to attacks. The condition (9) guarantees that attacks happen rarely on average. Note also that when $N\left(k_{2}, k_{1}\right)$ is defined to denote the number of packet exchange failures due to attacks over all packet exchange attempts at times $\tau_{k_{1}}, \tau_{k_{1}+1}, \ldots, \tau_{k_{2}-1}$, 
(10) implies (9). Specifically, (10) reduces to (9) by setting $N\left(k_{2}, k_{1}\right) \triangleq \sum_{i=k_{1}}^{k_{2}-1} l_{\mathrm{M}}(i), k_{1}=0$, and $k_{2}=k$.

As we have observed so far, the attack model in (9) is sufficiently general to cover known models. We further generalize it, because even though the model in (9) allows stochasticity in the generation of $l_{\mathrm{M}}(\cdot)$, it is not enough to characterize certain stochastic attacks. An example is the case where each packet exchange attempt faces an attack with a fixed probability (e.g., $\left\{l_{\mathrm{M}}(i) \in\{0,1\}\right\}_{i \in \mathbb{N}_{0}}$ is a Bernoulli process). To cover such stochastic attacks as well as attacks characterized in (9), we consider a model where $\left\{l_{\mathrm{M}}(i) \in\{0,1\}\right\}_{i \in \mathbb{N}_{0}}$ is given through conditions similar to (4). Specifically, we assume that there exists a scalar $\rho_{\mathrm{M}} \in[0,1]$ such that

$$
\sum_{k=1}^{\infty} \mathbb{P}\left[\sum_{i=0}^{k-1} l_{\mathrm{M}}(i)>\rho_{\mathrm{M}} k\right]<\infty .
$$

The following lemma shows that the characterization with 11 is more general than the one provided by 9 .

Lemma 2.3: Suppose the binary-valued process $\left\{l_{\mathrm{M}}(i) \in\right.$ $\{0,1\}\}_{i \in \mathbb{N}_{0}}$ satisfies (9) with $\kappa \geq 0$ and $\tau>1$. Then (11) holds for all $\rho_{\mathrm{M}} \in\left(\frac{1}{\tau}, 1\right)$.

Proof: Using Markov's inequality we obtain

$$
\begin{aligned}
& \mathbb{P}\left[\sum_{i=0}^{k-1} l_{\mathrm{M}}(i)>\rho_{\mathrm{M}} k\right] \leq \mathbb{P}\left[\sum_{i=0}^{k-1} l_{\mathrm{M}}(i) \geq \rho_{\mathrm{M}} k\right] \\
& \quad=\mathbb{P}\left[e^{\sum_{i=0}^{k-1} l_{\mathrm{M}}(i)} \geq e^{\rho_{\mathrm{M}} k}\right] \leq e^{-\rho_{\mathrm{M}} k} \mathbb{E}\left[e^{\sum_{i=0}^{k-1} l_{\mathrm{M}}(i)}\right]
\end{aligned}
$$

for $k \in \mathbb{N}$. By $(9)$, we have $\mathbb{E}\left[e^{\sum_{i=0}^{k-1} l_{\mathrm{M}}(i)}\right] \leq \mathbb{E}\left[e^{\kappa+\frac{k}{\tau}}\right]=$ $e^{\kappa+\frac{k}{\tau}}$. Therefore, it follows from (12) that $\mathbb{P}\left[\sum_{i=0}^{k-1} l_{\mathrm{M}}(i)>\right.$ $\left.\rho_{\mathrm{M}} k\right] \leq e^{\kappa-\left(\rho_{\mathrm{M}}-\frac{1}{\tau}\right) k}, k \in \mathbb{N}$. Thus, for all $\rho_{\mathrm{M}} \in\left(\frac{1}{\tau}, 1\right)$,

$$
\begin{aligned}
\sum_{k=1}^{\infty} & \mathbb{P}\left[\sum_{i=0}^{k-1} l_{\mathrm{M}}(i)>\rho_{\mathrm{M}} k\right] \leq \sum_{k=1}^{\infty} e^{\kappa-\left(\rho_{\mathrm{M}}-\frac{1}{\tau}\right) k} \\
= & e^{\kappa} e^{-\left(\rho_{\mathrm{M}}-\frac{1}{\tau}\right)}\left(1-e^{-\left(\rho_{\mathrm{M}}-\frac{1}{\tau}\right)}\right)^{-1}<\infty
\end{aligned}
$$

which completes the proof.

Thus, if the only cause of packet losses is attacks (i.e., $l(i)=$ $l_{\mathrm{M}}(i)$ ), then Assumption 2.1 holds with $\rho=\rho_{\mathrm{M}}$.

3) Combination of Random and Malicious Packet Losses (independent case): In order to model the case where the network is subject to both random and malicious packet losses, we define $\{l(i) \in\{0,1\}\}_{i \in \mathbb{N}_{0}}$ by

$$
l(i)= \begin{cases}1, & l_{\mathrm{R}}(i)=1 \text { or } l_{\mathrm{M}}(i)=1, \quad i \in \mathbb{N}_{0}, \\ 0, & \text { otherwise }\end{cases}
$$

where $\left\{l_{\mathrm{R}}(i) \in\{0,1\}\right\}_{i \in \mathbb{N}_{0}}$ is a time-inhomogeneous Markov chain given in (5) characterizing random packet losses (from Section II-B1) and $\left\{l_{\mathrm{M}}(i) \in\{0,1\}\right\}_{i \in \mathbb{N}_{0}}$ satisfying (11) is a binary-valued process that represents attacks of a malicious agent (from Section II-B2).

Proposition 2.4 below provides a range of values for $\rho \in(0,1)$ that satisfy Assumption 2.1 in the case where the network faces both random and malicious packet losses.

Proposition 2.4: Consider the packet exchange failure indicator process $\{l(i) \in\{0,1\}\}_{i \in \mathbb{N}_{0}}$ given by (13) where
$\left\{l_{\mathrm{R}}(i) \in\{0,1\}\right\}_{i \in \mathbb{N}_{0}}$ and $\left\{l_{\mathrm{M}}(i) \in\{0,1\}\right\}_{i \in \mathbb{N}_{0}}$ are mutually independent. Assume

$$
p_{1}+p_{0} \rho_{\mathrm{M}}<1
$$

where $p_{1}, p_{0} \in(0,1)$ are scalars that satisfy (6), (7). Then (4) holds for all $\rho \in\left(p_{1}+p_{0} \rho_{\mathrm{M}}, 1\right)$.

Proof: From (13), the overall loss process can be given by

$$
l(i)=l_{\mathrm{R}}(i)+\left(1-l_{\mathrm{R}}(i)\right) l_{\mathrm{M}}(i), \quad i \in \mathbb{N}_{0},
$$

and hence, by (3),

$$
L(k)=\sum_{i=0}^{k-1} l_{\mathrm{R}}(i)+\sum_{i=0}^{k-1}\left(1-l_{\mathrm{R}}(i)\right) l_{\mathrm{M}}(i), \quad k \in \mathbb{N} .
$$

Now, let $\epsilon \triangleq \rho-p_{1}-p_{0} \rho_{\mathrm{M}}, \epsilon_{2} \triangleq \min \left\{\frac{\epsilon}{2}, \frac{\rho_{\mathrm{M}}-p_{0} \rho_{\mathrm{M}}}{2}\right\}, \epsilon_{1} \triangleq$ $\epsilon-\epsilon_{2}$, and define $\rho_{1} \triangleq p_{1}+\epsilon_{1}, \rho_{2} \triangleq p_{0} \rho_{\mathrm{M}}+\epsilon_{2}$. Furthermore, let $L_{1}(k) \triangleq \sum_{i=0}^{k-1} l_{\mathrm{R}}(i)$ and $L_{2}(k) \triangleq \sum_{i=0}^{k-1}\left(1-l_{\mathrm{R}}(i)\right) l_{\mathrm{M}}(i)$. We then have

$$
\begin{aligned}
\mathbb{P}[L(k)>\rho k] & =\mathbb{P}\left[L_{1}(k)+L_{2}(k)>\rho_{1} k+\rho_{2} k\right] \\
& \leq \mathbb{P}\left[\left\{L_{1}(k)>\rho_{1} k\right\} \cup\left\{L_{2}(k)>\rho_{2} k\right\}\right] \\
& \leq \mathbb{P}\left[L_{1}(k)>\rho_{1} k\right]+\mathbb{P}\left[L_{2}(k)>\rho_{2} k\right] .
\end{aligned}
$$

In the following we will show that the series $\sum_{k=1}^{\infty} \mathbb{P}\left[L_{1}(k)>\right.$ $\left.\rho_{1} k\right]$ and $\sum_{k=1}^{\infty} \mathbb{P}\left[L_{2}(k)>\rho_{2} k\right]$ are convergent.

First, note that

$$
\begin{aligned}
\rho_{1} & =p_{1}+\epsilon-\epsilon_{2}=\max \left\{p_{1}+\frac{\epsilon}{2}, p_{1}+\epsilon-\frac{\rho_{\mathrm{M}}-p_{0} \rho_{\mathrm{M}}}{2}\right\} \\
& =\max \left\{\frac{p_{1}+\rho-p_{0} \rho_{\mathrm{M}}}{2}, \rho-p_{0} \rho_{\mathrm{M}}-\frac{\rho_{\mathrm{M}}-p_{0} \rho_{\mathrm{M}}}{2}\right\} \\
& =\max \left\{\frac{p_{1}+\rho-p_{0} \rho_{\mathrm{M}}}{2}, \frac{2 \rho-\rho_{\mathrm{M}}\left(1+p_{0}\right)}{2}\right\}
\end{aligned}
$$

As $\frac{p_{1}+\rho-p_{0} \rho_{\mathrm{M}}}{2}<1$ and $\frac{2 \rho-\rho_{\mathrm{M}}\left(1+p_{0}\right)}{2}<1$, it holds from 17 that $\rho_{1} \in\left(p_{1}, 1\right)$. Consequently, $\sum_{k=1}^{\infty} \mathbb{P}\left[L_{1}(k)>\rho_{1} k\right]<\infty$ follows from Lemma 2.1 with $\rho_{\mathrm{R}}$ replaced with $\rho_{1}$.

Next, we will use Lemma A.1 to show that $\sum_{k=1}^{\infty} \mathbb{P}\left[L_{2}(k)>\rho_{2} k\right]<\infty$. To obtain this result, we first observe that $\rho_{2}>p_{0} \rho_{\mathrm{M}}$, since $\epsilon_{2}>0$. Moreover,

$$
\begin{aligned}
\rho_{2} & =p_{0} \rho_{\mathrm{M}}+\min \left\{\frac{\epsilon}{2}, \frac{\rho_{\mathrm{M}}-p_{0} \rho_{\mathrm{M}}}{2}\right\} \leq p_{0} \rho_{\mathrm{M}}+\frac{\rho_{\mathrm{M}}-p_{0} \rho_{\mathrm{M}}}{2} \\
& <p_{0} \rho_{\mathrm{M}}+\rho_{\mathrm{M}}-p_{0} \rho_{\mathrm{M}}=\rho_{\mathrm{M}},
\end{aligned}
$$

and hence, we have $\rho_{2} \in\left(p_{0} \rho_{\mathrm{M}}, \rho_{\mathrm{M}}\right)$. As a consequence of (9), conditions 67, 68 in the Lemma A.1 hold with $\tilde{p}=p_{0}$ and $\tilde{w}=\rho_{\mathrm{M}}$, together with processes $\{\xi(i) \in\{0,1\}\}_{i \in \mathbb{N}_{0}}$ and $\{\chi(i) \in\{0,1\}\}_{i \in \mathbb{N}_{0}}$ defined by setting $\xi(i)=1-l_{\mathrm{R}}(i)$, $\chi(i)=l_{\mathrm{M}}(i), i \in \mathbb{N}_{0}$. Now, we have $L_{2}(k)=\sum_{i=0}^{k-1} \xi(i) \chi(i)$ and hence, Lemma A.1 implies $\sum_{k=1}^{\infty} \mathbb{P}\left[L_{2}(k)>\rho_{2} k\right]<\infty$.

Finally, by (16), we arrive at

$$
\begin{aligned}
& \sum_{k=1}^{\infty} \mathbb{P}[L(k)>\rho k] \\
& \quad \leq \sum_{k=1}^{\infty} \mathbb{P}\left[L_{1}(k)>\rho_{1} k\right]+\sum_{k=1}^{\infty} \mathbb{P}\left[L_{2}(k)>\rho_{2} k\right]<\infty,
\end{aligned}
$$

which completes the proof. 
4) Combination of Random and Malicious Packet Losses (dependent case): So far, in Proposition 2.4 we assumed that packet exchange attempt failures due to attacks are independent of those due to random packet losses. Next, we consider the case where the two processes $\left\{l_{\mathrm{R}}(i)\right\}_{i \in \mathbb{N}_{0}}$ and $\left\{l_{\mathrm{M}}(i)\right\}_{i \in \mathbb{N}_{0}}$ may be dependent. This is clearly the case when the attacker has information of the random packet losses in the channel. Furthermore, as we discussed in the Introduction, the attacker may decide to attack based on the content of packets. In such cases $l_{\mathrm{M}}(\cdot)$ would depend on state and control input, which in turn depend on $l_{\mathrm{R}}(\cdot)$. Proposition 2.5 below deals with such cases.

Proposition 2.5: Consider the packet exchange failure indicator process $\{l(i) \in\{0,1\}\}_{i \in \mathbb{N}_{0}}$. Assume

$$
p_{1}+\rho_{\mathrm{M}}<1,
$$

where $p_{1} \in(0,1)$ is a scalar that satisfies (6). Then (4) holds for all $\rho \in\left(p_{1}+\rho_{\mathrm{M}}, 1\right)$.

Proof: It follows from (13) that

$$
L(k) \leq \sum_{i=0}^{k-1} l_{\mathrm{R}}(i)+\sum_{i=0}^{k-1} l_{\mathrm{M}}(i), \quad k \in \mathbb{N} .
$$

Now, using arguments similar to the ones used for obtaining (16) in the proof of Proposition 2.4, we have

$$
\begin{aligned}
& \mathbb{P}[L(k)>\rho k] \leq \mathbb{P}\left[\sum_{i=0}^{k-1} l_{\mathrm{R}}(i)+\sum_{i=0}^{k-1} l_{\mathrm{M}}(i)>\rho k\right] \\
& \leq \mathbb{P}\left[\sum_{i=0}^{k-1} l_{\mathrm{R}}(i)>\rho_{1} k\right]+\mathbb{P}\left[\sum_{i=0}^{k-1} l_{\mathrm{M}}(i)>\rho_{2} k\right], k \in \mathbb{N},
\end{aligned}
$$

and consequently

$$
\begin{aligned}
& \sum_{k=1}^{\infty} \mathbb{P}[L(k)>\rho k] \\
& \leq \sum_{k=1}^{\infty} \mathbb{P}\left[\sum_{i=0}^{k-1} l_{\mathrm{R}}(i)>\rho_{1} k\right]+\sum_{k=1}^{\infty} \mathbb{P}\left[\sum_{i=0}^{k-1} l_{\mathrm{M}}(i)>\rho_{2} k\right],
\end{aligned}
$$

where $\rho_{1} \triangleq p_{1}+\frac{\epsilon}{2}, \rho_{2} \triangleq \rho_{\mathrm{M}}+\frac{\epsilon}{2}$, and $\epsilon \triangleq \rho-p_{1}-\rho_{\mathrm{M}}$.

Observe that $\rho_{1}=p_{1}+\frac{\rho-p_{1}-\rho_{\mathrm{M}}}{2}=\frac{\rho+p_{1}-\rho_{\mathrm{M}}}{2}$. Since $\frac{\rho+p_{1}-\rho_{\mathrm{M}}}{2}<1$ and $\epsilon>0$, we have $\rho_{1} \in\left(p_{1}, 1\right)$. By using Lemma 2.1 with $\rho_{\mathrm{R}}=\rho_{1}$, we obtain

$$
\sum_{k=1}^{\infty} \mathbb{P}\left[L_{1}(k)>\rho_{1} k\right]<\infty
$$

Furthermore, note that $\rho_{2}=\rho_{\mathrm{M}}+\frac{\rho-p_{1}-\rho_{\mathrm{M}}}{2}=\frac{\rho+\rho_{\mathrm{M}}-p_{1}}{2}$. Also, by $\frac{\rho+\rho_{\mathrm{M}}-p_{1}}{2}<1$ and $\epsilon>0$, we have $\rho_{2} \in\left(\rho_{\mathrm{M}}, 1\right)$. Since $\rho_{2}>\rho_{\mathrm{M}}$, by the characterization of $\left\{l_{\mathrm{M}}(i) \in\{0,1\}\right\}_{i \in \mathbb{N}_{0}}$,

$$
\sum_{k=1}^{\infty} \mathbb{P}\left[\sum_{i=0}^{k-1} l_{\mathrm{M}}(i)>\rho_{2} k\right] \leq \sum_{k=1}^{\infty} \mathbb{P}\left[\sum_{i=0}^{k-1} l_{\mathrm{M}}(i)>\rho_{\mathrm{M}} k\right]<\infty .
$$

The result then follows from (20)-(22).

In comparison with Proposition 2.4, the result above provides a more restricted range of values for $\rho$ that satisfies Assumption 2.1 This is because in Proposition 2.5 we find $\rho$ for the worst case scenario where the attacker may be knowledgeable about all random packet losses in the network and may have access to the information of the transmitted state and control input vectors. An example scenario is where the attacker avoids placing malicious attacks when there is already a random packet loss, increasing the total number of packet exchange failures, which is clearly to the disadvantage of the controller to maintain closed-loop stability.

We note that the condition (18) guarantees that the range $\rho \in\left(p_{1}+\rho_{\mathrm{M}}, 1\right)$ identified in Proposition 2.5 is well defined. If $p_{1}+\rho_{M} \geq 1$, then Assumption 2.1 holds with $\rho=1$. We also note that Proposition 2.5 may introduce some conservativeness when it is applied to other scenarios where malicious attacks and random packet losses are dependent, but not as in the worst case scenario mentioned above. In such cases additional information about the malicious attacks and random packet losses may be employed to show that Assumption 2.1 holds with $\rho<1$ even if $p_{1}+\rho_{\mathrm{M}} \geq 1$.

Remark 2.6: There may be situations where the attacker has limited knowledge. For instance, the attacker may have access only to certain entries of the state and control input vectors. This situation arises in a multi-hop network with multiple paths (see, e.g., [16], [45]); different parts of the state and control input vectors may be sent over different paths on the network and the attacker may have access to the data only on some of those paths. In this case the attacker would need an estimation mechanism to have information about the state/control input vectors. Note that the operator may also utilize encryption methods to prevent the attacker gain any information about the system behavior. In the situations where the attacker is not knowledgeable about the random packet losses and has no information of state and control input vectors, Proposition 2.4 can be used.

\section{Event-Triggered Control Design}

In this section we investigate event-triggered control of (1) over an unreliable and potentially attacked network characterized through Assumption 2.1

As a first step, we introduce the event-triggering scheme for communication between the plant and the controller. This scheme will determine the time instants $\tau_{i} \in \mathbb{N}_{0}, i \in \mathbb{N}_{0}$, at which packet exchanges are attempted. For this purpose, we utilize the quadratic Lyapunov-like function $V: \mathbb{R}^{n} \rightarrow[0, \infty)$ given by $V(x) \triangleq x^{\mathrm{T}} P x$, where $P>0$. Letting $\tau_{0}=0$, we describe $\tau_{i}, i \in \mathbb{N}$, by

$$
\begin{array}{r}
\tau_{i+1} \triangleq \min \left\{t \in\left\{\tau_{i}+1, \tau_{i}+2, \ldots\right\}: t \geq \tau_{i}+\theta\right. \\
\text { or } \left.V\left(A x(t)+B u\left(\tau_{i}\right)\right)>\beta V\left(x\left(\tau_{i}\right)\right)\right\},
\end{array}
$$

where $\beta \in(0,1), \theta \in \mathbb{N}$.

The triggering condition (23) involves two parts. The part $V\left(A x(t)+B u\left(\tau_{i}\right)\right)>\beta V\left(x\left(\tau_{i}\right)\right)$ ensures that after a successful packet exchange attempt at $\tau_{i}$, the value of $V(\cdot)$ stays below the level $\beta V\left(x\left(\tau_{i}\right)\right)$ until the next packet exchange attempt. Furthermore, the triggering condition $t \geq \tau_{i}+\theta$ ensures that two consecutive packet exchange attempt instants are at most $\theta \in \mathbb{N}$ steps apart, that is, $\tau_{i+1}-\tau_{i} \leq \theta, i \in \mathbb{N}_{0}$. Although the specific value of $\theta$ does not affect the results 


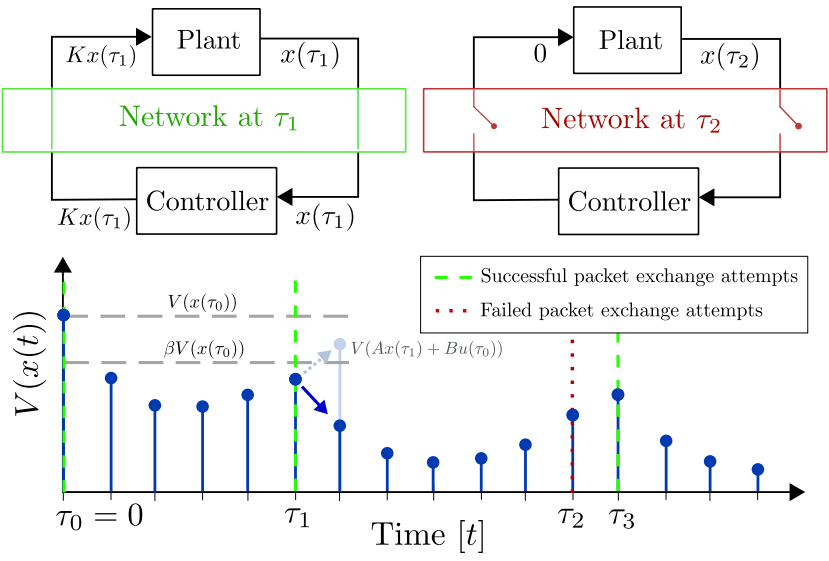

Figure 1. [Top] Networked control system with successful (left) and failed (right) packet transmissions.

[Bottom] Response of the Lyapunov-like function.

developed below, the boundedness of packet exchange attempt intervals guarantees that $\tau_{i}$ (and hence $V\left(x\left(\tau_{i}\right)\right)$ ) is welldefined for each $i \in \mathbb{N}$. In practice, the value of $\theta$ can be selected considering how frequent the plant state is desired to be monitored by the controller side.

The operation of the event-triggered networked control system is illustrated in Fig. 11 The triggering condition (23) is checked at the plant side at each step $t \in \mathbb{N}_{0}$. At times $t=\tau_{i}, i \in \mathbb{N}$, the triggering condition is satisfied and packet exchanges are attempted. In this example, a packet exchange is attempted at time $t=\tau_{1}$, since $V\left(A x(t)+B u\left(\tau_{0}\right)\right)>$ $\beta V\left(x\left(\tau_{0}\right)\right)$. At this time instant, the plant and the controller successfully exchange state and control input packets over the network, and as a result, control input on the plant side is updated to $K x\left(\tau_{1}\right)$. Note that packet exchange attempts are not always successful, and may fail due to loss of packets in the network. In the figure, the packet exchange attempt at time $\tau_{2}$ fails. In this case, it follows from (2) with $l(2)=1$ that the control input at the plant side is set to 0 at time $\tau_{2}$, which results in an unstable behavior. A packet exchange is attempted again at the very next time step $\tau_{3}$, since the triggering condition is also satisfied at that time instant.

\section{A. Stability Analysis}

Next, we investigate stability of the closed-loop eventtriggered networked control system (1), (2), (23), which is a stochastic dynamical system due to the probabilistic characterization of packet losses. Below we define almost sure asymptotic stability for stochastic dynamical systems.

Definition 3.1: The zero solution $x(t) \equiv 0$ of the stochastic system (1), (2), and (23) is almost surely stable if, for all $\epsilon>0$ and $\bar{p}>0$, there exists $\delta=\delta(\epsilon, \bar{p})>0$ such that if $\|x(0)\|<\delta$, then

$$
\mathbb{P}\left[\sup _{t \in \mathbb{N}_{0}}\|x(t)\|>\epsilon\right]<\bar{p} .
$$

Moreover, the zero solution $x(t) \equiv 0$ is asymptotically stable almost surely if it is almost surely stable and

$$
\mathbb{P}\left[\lim _{t \rightarrow \infty}\|x(t)\|=0\right]=1 .
$$

In our stability analysis for the networked control system (1), (2), we utilize an upper bound for the long run average of the total number of failed packet exchanges. The following result is a direct consequence of the Borel-Cantelli lemma (see [46]) and shows that under Assumption 2.1, the long run average of the total number of failed packet exchanges is upper bounded by $\rho$ characterized in (4).

Lemma 3.2: If there exists a scalar $\rho \in[0,1]$ such that (4) holds, then

$$
\limsup _{k \rightarrow \infty} \frac{L(k)}{k} \leq \rho,
$$

almost surely.

In Propositions 2.4 and 2.5, we obtained a range of values for $\rho$ that satisfy (4). In those results the range was given as an open interval. In the following result we show that when Assumption 2.1 holds for a range of values, then (26) also holds with $\rho$ given as the infimum of the range.

Lemma 3.3: Suppose (4) is satisfied for all $\rho \in(\rho, 1)$ where $\rho \in[0,1)$. Then (26) holds with $\rho=\rho$, almost surely.

Proof: The proof resembles the sufficiency part of the proof of Proposition 5.6 in [47]. First, by Lemma 3.2.

$$
\mathbb{P}\left[\limsup _{k \rightarrow \infty} \frac{L(k)}{k}-\underline{\rho}>\epsilon\right]=0,
$$

for any $\epsilon>0$. Now, it follows from (27) that

$$
\begin{aligned}
& \mathbb{P}\left[\limsup _{k \rightarrow \infty} \frac{L(k)}{k}-\underline{\rho}>0\right]=\mathbb{P}\left[\cup_{j=1}^{\infty}\left\{\limsup _{k \rightarrow \infty} \frac{L(k)}{k}-\underline{\rho}>\frac{1}{j}\right\}\right] \\
& \leq \sum_{j=1}^{\infty} \mathbb{P}\left[\limsup _{k \rightarrow \infty} \frac{L(k)}{k}-\underline{\rho}>\frac{1}{j}\right]=0,
\end{aligned}
$$

which implies that $\mathbb{P}\left[\lim \sup _{k \rightarrow \infty} \frac{L(k)}{k} \leq \underline{\rho}\right]=1$.

Remark 3.4: Note that the term $\lim \sup _{k \rightarrow \infty} \frac{L(k)}{k}$ in (26) corresponds to the "discrete event rate" used in [48], [49] for deterministic systems, when $\lim _{k \rightarrow \infty} \frac{L(k)}{k}$ exists. In this paper, Assumption 2.1 allows the binary-valued process $\{l(i) \in$ $\{0,1\}\}_{i \in \mathbb{N}_{0}}$ to be a non-ergodic stochastic process, for which $\lim _{k \rightarrow \infty} \frac{L(k)}{k}$ may not be equal for all sample paths. For instance, let $l(i) \triangleq l_{\mathrm{M}}(i), i \in \mathbb{N}_{0}$, and

$$
l_{\mathrm{M}}(i) \triangleq \begin{cases}1, & i \in\{\alpha, 2 \alpha, 3 \alpha \ldots\}, \\ 0, & \text { otherwise }\end{cases}
$$

where $\alpha: \Omega \rightarrow\{2,4\}$ is a random variable with $\mathbb{P}[\alpha=2]=$ $\mathbb{P}[\alpha=4]=\frac{1}{2}$. In this setting, the attacker decides the period of attacks based on a random variable $\alpha: \Omega \rightarrow\{2,4\}$. Depending on the value of $\alpha$, malicious packet losses occur either at every 2 packet exchange attempts or at every 4 packet exchange attempts. Thus, the discrete event rate would be a random variable that depends on the value of $\alpha$. On the other hand, regardless of the value of $\alpha,(9)$ is satisfied with $\tau=2$, and hence Lemmas 2.3 and 3.3 imply that $\limsup _{k \rightarrow \infty} \frac{L(k)}{k} \leq \frac{1}{2}$, almost surely. Note that here $\frac{1}{2}$ represents the worst-case upper bound for the long run average of the total number of failed packet exchanges.

We are now ready to state the main result of this paper. It provides a sufficient condition for almost sure asymptotic 
stability of the networked control system (1), (2) with packet exchange failure indicator $\{l(i) \in\{0,1\}\}_{i \in \mathbb{N}_{0}}$ satisfying (26).

Theorem 3.5: Consider the linear dynamical system (1). Suppose that the process $\{l(i) \in\{0,1\}\}_{i \in \mathbb{N}_{0}}$ characterizing packet exchange failures 1 in the network satisfies 26 with scalar $\rho \in[0,1]$. If there exist a matrix $K \in \mathbb{R}^{m \times n}$, a positivedefinite matrix $P \in \mathbb{R}^{n \times n}$, and scalars $\beta \in(0,1), \varphi \in[1, \infty)$ such that

$$
\begin{aligned}
& (A+B K)^{\mathrm{T}} P(A+B K)-\beta P \leq 0, \\
& A^{\mathrm{T}} P A-\varphi P \leq 0, \\
& (1-\rho) \ln \beta+\rho \ln \varphi<0,
\end{aligned}
$$

then the event-triggered control law (2), (23) guarantees almost sure asymptotic stability of the zero solution $x(t) \equiv 0$ of the closed-loop system dynamics.

Proof: The proof is composed of three steps. In the initial step, we obtain an inequality concerning the evolution of the Lyapunov-like function $V(x) \triangleq x^{\mathrm{T}} P x, x \in \mathbb{R}^{n}$. Then, we will establish almost sure stability, and then finally we show almost sure asymptotic stability of the closed-loop system.

First, we use (1) and (2) together with $V(\cdot)$ to obtain

$$
\begin{aligned}
V\left(x\left(\tau_{i}+1\right)\right)= & x^{\mathrm{T}}\left(\tau_{i}\right)(A+(1-l(i)) B K)^{\mathrm{T}} P \\
& \cdot(A+(1-l(i)) B K) x\left(\tau_{i}\right), i \in \mathbb{N}_{0} .
\end{aligned}
$$

Now, for the case $l(i)=0,(28)$ and (31) imply

$$
\begin{aligned}
V\left(x\left(\tau_{i}+1\right)\right) & =x^{\mathrm{T}}\left(\tau_{i}\right)(A+B K)^{\mathrm{T}} P(A+B K) x\left(\tau_{i}\right) \\
& \leq \beta x^{\mathrm{T}}\left(\tau_{i}\right) \operatorname{Px}\left(\tau_{i}\right) .
\end{aligned}
$$

Since $\tau_{i+1} \geq \tau_{i}+1$, it follows from (23) and (32) that

$$
\begin{aligned}
V(x(t)) & \leq \beta x^{\mathrm{T}}\left(\tau_{i}\right) \operatorname{Px}\left(\tau_{i}\right) \\
& =\beta V\left(x\left(\tau_{i}\right)\right), \quad t \in\left\{\tau_{i}+1, \ldots, \tau_{i+1}\right\} .
\end{aligned}
$$

On the other hand, for the case $l(i)=1$, we have from (29) and (31) that

$$
V\left(x\left(\tau_{i}+1\right)\right)=x^{\mathrm{T}}\left(\tau_{i}\right) A^{\mathrm{T}} P A x\left(\tau_{i}\right) \leq \varphi x^{\mathrm{T}}\left(\tau_{i}\right) P x\left(\tau_{i}\right) .
$$

Now if $\tau_{i+1}=\tau_{i}+1$, we have $V\left(x\left(\tau_{i+1}\right)\right) \leq \varphi V\left(x\left(\tau_{i}\right)\right)$ due to (34). Otherwise, that is, if $\tau_{i+1}>\tau_{i}+1$, it means that $V(x(t)) \leq \beta V\left(x\left(\tau_{i}\right)\right)$ for $t \in\left\{\tau_{i}+2, \ldots, \tau_{i+1}\right\}$. Therefore, since $\beta \leq \varphi$,

$$
V(x(t)) \leq \varphi V\left(x\left(\tau_{i}\right)\right), \quad t \in\left\{\tau_{i}+1, \ldots, \tau_{i+1}\right\} .
$$

Using (33) and (35) we obtain

$$
V\left(x\left(\tau_{i+1}\right)\right) \leq(1-l(i)) \beta V\left(x\left(\tau_{i}\right)\right)+l(i) \varphi V\left(x\left(\tau_{i}\right)\right),
$$

for $i \in \mathbb{N}_{0}$. Note that the inequality given in (36) provides an upper bound on $V(\cdot)$.

Now, let $\eta(k) \triangleq \prod_{i=0}^{k-1}[(1-l(i)) \beta+l(i) \varphi]$. Then, by (36),

$$
V\left(x\left(\tau_{k}\right)\right) \leq \eta(k) V(x(0)), \quad k \in \mathbb{N} .
$$

\footnotetext{
${ }^{1}$ We set 26 as a condition for packet exchange failures as it allows more generality in comparison to Assumption 2.1 Note that by Lemma 3.2 Assumption 2.1 implies 26. Furthermore, Lemma 3.3 shows that 26] also holds when $\rho$ is given as the infimum of an open interval where all values satisfy Assumption 2.1
}

Furthermore, since $\ln [(1-q) \beta+q \varphi]=(1-q) \ln \beta+q \ln \varphi$ for $q \in\{0,1\}$, we have

$$
\begin{aligned}
\ln \eta(k) & =\sum_{i=0}^{k-1} \ln [(1-l(i)) \beta+l(i) \varphi] \\
& =\sum_{i=0}^{k-1}(1-l(i)) \ln \beta+\sum_{i=0}^{k-1} l(i) \ln \varphi \\
& =(k-L(k)) \ln \beta+L(k) \ln \varphi,
\end{aligned}
$$

where $L(k)=\sum_{i=0}^{k-1} l(i)$ by (3). Now by $\beta \in(0,1)$, and $\varphi \in[1, \infty)$, it follows from (26) and (30) that

$$
\begin{aligned}
\limsup _{k \rightarrow \infty} \frac{\ln \eta(k)}{k} & =\limsup _{k \rightarrow \infty} \frac{1}{k}[(k-L(k)) \ln \beta+L(k) \ln \varphi] \\
& \leq(1-\rho) \ln \beta+\rho \ln \varphi<0,
\end{aligned}
$$

almost surely. As a consequence, $\lim _{k \rightarrow \infty} \ln \eta(k)=-\infty$, and hence, $\lim _{k \rightarrow \infty} \eta(k)=0$, almost surely. Thus, for any $\epsilon>0$, $\lim _{j \rightarrow \infty} \mathbb{P}\left[\sup _{k \geq j} \eta(k)>\epsilon^{2}\right]=0$. Therefore, for any $\epsilon>0$ and $\bar{p}>0$, there exists a positive integer $N(\epsilon, \bar{p})$ such that

$$
\mathbb{P}\left[\sup _{k \geq j} \eta(k)>\epsilon^{2}\right]<\bar{p}, \quad j \geq N(\epsilon, \bar{p}) .
$$

In what follows, we employ (37) and (38) to show almost sure stability of the closed-loop system. Note that (33), (35), and $\varphi \geq 1>\beta$ imply that $V(x(t+1)) \leq \varphi V(x(t)), t \in$ $\left\{\tau_{i}, \ldots, \tau_{i+1}-1\right\}, i \in \mathbb{N}_{0}$. Since $\|x\|^{2} \leq \frac{1}{\lambda_{\min }(P)} V(x)$ and $V(x) \leq \lambda_{\max }(P)\|x\|^{2}, x \in \mathbb{R}^{n}$, we have

$$
\|x(t)\|^{2} \leq \varphi \nu\left\|x\left(\tau_{i}\right)\right\|^{2}, t \in\left\{\tau_{i}, \ldots, \tau_{i+1}-1\right\}
$$

for $i \in \mathbb{N}_{0}$, where $\nu \triangleq \frac{\lambda_{\max }(P)}{\lambda_{\min }(P)}$.

Now, let $\mathcal{T}_{k} \triangleq\left\{\tau_{k}, \ldots, \tau_{k+1}-1\right\}, k \in \mathbb{N}_{0}$. Then by using (37) and [39), we obtain $\eta(k) \geq \frac{V\left(x\left(\tau_{k}\right)\right)}{V(x(0))} \geq$ $\frac{\lambda_{\min }(P)}{\lambda_{\max }(P)} \frac{\left\|x\left(\tau_{k}\right)\right\|^{2}}{\|x(0)\|^{2}} \geq \frac{1}{\nu^{2} \varphi} \frac{\|x(t)\|^{2}}{\|x(0)\|^{2}}$ for all $t \in \mathcal{T}_{k}, k \in \mathbb{N}$. Hence, $\eta(k) \geq \frac{1}{\nu^{2} \varphi} \frac{\max _{t \in \mathcal{T}_{k}}\|x(t)\|^{2}}{\|x(0)\|^{2}}, k \in \mathbb{N}$. By (38), it follows that for all $\epsilon>0$ and $\bar{p}>0$,

$$
\begin{aligned}
& \mathbb{P}\left[\sup _{k \geq j} \max _{t \in \mathcal{T}_{k}}\|x(t)\|>\epsilon \nu \sqrt{\varphi}\|x(0)\|\right] \\
& \quad=\mathbb{P}\left[\sup _{k \geq j} \max _{t \in \mathcal{T}_{k}}\|x(t)\|^{2}>\epsilon^{2} \nu^{2} \varphi\|x(0)\|^{2}\right] \\
& \quad=\mathbb{P}\left[\sup _{k \geq j} \frac{1}{\nu^{2} \varphi} \frac{\max _{t \in \mathcal{T}_{k}}\|x(t)\|^{2}}{\|x(0)\|^{2}}>\epsilon^{2}\right] \\
& \quad \leq \mathbb{P}\left[\sup _{k \geq j} \eta(k)>\epsilon^{2}\right]<\bar{p}, \quad j \geq N(\epsilon, \bar{p}) .
\end{aligned}
$$

We now define $\delta_{1} \triangleq \frac{1}{\nu \sqrt{\varphi}}$. Note that if $\|x(0)\| \leq \delta_{1}$, then (since $\nu \sqrt{\varphi}\|x(0)\| \leq 1$ ) for all $j \geq N(\epsilon, \bar{p})$, we have

$$
\begin{aligned}
& \mathbb{P}\left[\sup _{k \geq j} \max _{t \in \mathcal{T}_{k}}\|x(t)\|>\epsilon\right] \\
& \quad \leq \mathbb{P}\left[\sup _{k \geq j} \max _{t \in \mathcal{T}_{k}}\|x(t)\|>\epsilon \nu \sqrt{\varphi}\|x(0)\|\right]<\bar{p} .
\end{aligned}
$$

On the other hand, since $\varphi \geq 1>\beta$, it follows from (36) that $V\left(x\left(\tau_{k}\right)\right) \leq \varphi^{k} V(x(0)) \leq \varphi^{N(\epsilon, \bar{p})-1} V(x(0))$ for all $k \in\{0,1, \ldots, N(\epsilon, \bar{p})-1\}$. Therefore, $\left\|x\left(\tau_{k}\right)\right\|^{2} \leq$ 
$\varphi^{N(\epsilon, \bar{p})-1} \frac{\lambda_{\max }(P)}{\lambda_{\min }(P)}\|x(0)\|^{2}=\varphi^{N(\epsilon, \bar{p})-1} \nu\|x(0)\|^{2}$. Furthermore, as a result of 39,

$$
\max _{t \in \mathcal{T}_{k}}\|x(t)\|^{2} \leq \varphi \nu\left\|x\left(\tau_{k}\right)\right\|^{2} \leq \nu^{2} \varphi^{N(\epsilon, \bar{p})}\|x(0)\|^{2},
$$

and hence, $\max _{t \in \mathcal{T}_{k}}\|x(t)\| \leq \nu \sqrt{\varphi^{N(\epsilon, \bar{p})}}\|x(0)\|$ for all $k \in\{0,1, \ldots, N(\epsilon, \bar{p})-1\}$. Let $\delta_{2} \triangleq \epsilon \nu^{-1} \sqrt{\varphi^{-N(\epsilon, \bar{p})}}$. Now, if $\|x(0)\| \leq \delta_{2}$, then $\max _{t \in \mathcal{T}_{k}}\|x(t)\| \leq \epsilon, k \in$ $\{0,1, \ldots, N(\epsilon, \bar{p})-1\}$, which implies

$$
\mathbb{P}\left[\max _{k \in\{0,1, \ldots, N(\epsilon, \bar{p})\}} \max _{t \in \mathcal{T}_{k}}\|x(t)\|>\epsilon\right]=0 .
$$

It follows from (40) and (41) that for all $\epsilon>0, \bar{p}>0$,

$$
\begin{gathered}
\mathbb{P}\left[\sup _{t \in \mathbb{N}_{0}}\|x(t)\|>\epsilon\right]=\mathbb{P}\left[\sup _{k \in \mathbb{N}_{0}} \max _{t \in \mathcal{T}_{k}}\|x(t)\|>\epsilon\right] \\
=\mathbb{P}\left[\left\{\max _{k \in\{0,1, \ldots, N(\epsilon, \bar{p})-1\}} \max _{t \in \mathcal{T}_{k}}\|x(t)\|>\epsilon\right\}\right. \\
\left.\cup\left\{\sup _{k \geq N(\epsilon, \bar{p})} \max _{t \in \mathcal{T}_{k}}\|x(t)\|>\epsilon\right\}\right] \\
\leq \mathbb{P}\left[\max _{k \in\{0,1, \ldots, N(\epsilon, \bar{p})-1\}} \max _{t \in \mathcal{T}_{k}}\|x(t)\|>\epsilon\right] \\
+\mathbb{P}\left[\sup _{k \geq N(\epsilon, \bar{p})} \max _{t \in \mathcal{T}_{k}}\|x(t)\|>\epsilon\right]<\bar{p},
\end{gathered}
$$

whenever $\|x(0)\|<\delta \triangleq \min \left(\delta_{1}, \delta_{2}\right)$, which implies almost sure stability.

Finally, in order to establish almost sure asymptotic stability of the zero solution, it remains to show 25. To this end, observe that $\mathbb{P}\left[\lim _{k \rightarrow \infty} \eta\left(\tau_{k}\right)=0\right]=1$. It follows from 37 that $\mathbb{P}\left[\lim _{k \rightarrow \infty} V\left(x\left(\tau_{k}\right)\right)=0\right]=1$, which implies (25). Hence the zero solution of the closed-loop system (11, (2), (23) is asymptotically stable almost surely.

Theorem 3.5 provides a sufficient condition under which the event-triggered control law (2), (23) guarantees almost sure asymptotic stability of the system (1) for the case of packet losses satisfying Assumption 2.1 Note that the scalars $\beta \in(0,1)$ and $\varphi \in[1, \infty)$ in conditions (28) and (29) characterize upper bounds on the growth of the Lyapunovlike function, and they are also related to closed-loop and open-loop bounds utilized in [36], [40]. Specifically, when a packet exchange attempt between the plant and the controller is successful at time $\tau_{i}$, the condition (28) together with (23) guarantees that $V\left(x\left(\tau_{i+1}\right)\right) \leq \beta V\left(x\left(\tau_{i}\right)\right)$. On the other hand, if a packet exchange is unsuccessful at time $\tau_{i}$, it follows from (23) and (29) that $V\left(x\left(\tau_{i+1}\right)\right) \leq \varphi V\left(x\left(\tau_{i}\right)\right)$. If successful packet exchanges are sufficiently frequent such that (30) is satisfied, then the closed-loop stability is guaranteed.

We remark that the analysis for the closed-loop system stability in the proof above is technically involved partly due to the general characterization in Assumption 2.1, which captures not only random packet losses but attacks as well. If we consider only random packet losses, we may employ methods from discrete-time Markov jump systems theory [50] for obtaining conditions of stability. Furthermore, in the case $\{l(i) \in\{0,1\}\}_{i \in \mathbb{N}_{0}}$ is an ergodic process, the results presented in [40] can be directly employed to show stability.

On the other hand packet losses due to attacks (Section [I-B2 cannot be described using Markov processes and they may not be ergodic. Stability of a system under denialof-service attacks is explored in [10], where the analysis relies on a deterministic approach for obtaining an exponentially decreasing upper bound for the norm of the state. In contrast, in our analysis, we use probabilistic approaches similar to [23], [40] to show almost sure asymptotic stability. Specifically, we use tools from probability theory to find a stochastic upper bound for a Lyapunov-like function and show that this bound tends to zero even though it may increase at certain times. This approach is related to obtaining an upper bound on the top Lyapunov exponent (see [37]-[39]) of a stochastic system.

Theorem 3.5 provides conditions that guarantee both 24 and (25) implying almost sure asymptotic stability. In this stability definition, 25) is concerned with the convergence of solutions to zero, while (24) ensures that states sufficiently close to the origin are likely to stay close to the origin. However note that (24) allows states to leave any given ball in a finite time with positive (even if small) probability. For instance, if many consecutive packet transmission attempts fail, the state magnitude may grow due to lack of control action. We emphasize that Assumption 2.1 and hence (26) ensure packet failures to be statistically rare so that the state eventually converges to the origin.

Remark 3.6: In addition to almost sure stability, there are other stochastic stability and performance notions that are useful for the analysis of networked control systems. In particular, moment stability and moment-based performance notions have been utilized when random packet losses are considered (see [19], [20] and the references therein). In comparison with those works, in our problem setting, we must take into account also the effect of malicious attacks. We remark that in contrast with random packet losses, precise information of the probabilities of malicious attacks is not available. Hence, it is difficult to characterize the evolution of the moments of the state and establish moment stability. On the other hand, both random packet losses and malicious attacks, as well as their combination provide us information about the asymptotic ratio of packet exchange failures, which can be employed in the analysis when we consider almost sure asymptotic stability.

In the following corollary of Theorem 3.5, we discuss the special case of random packet losses described with timehomogeneous Markov chains.

Corollary 3.7: Consider the linear dynamical system (1). Suppose that the process $\{l(i) \in\{0,1\}\}_{i \in \mathbb{N}_{0}}$ is an irreducible time-homogeneous Markov chain with constant transition probabilities $p_{q, r} \in[0,1], q, r \in\{0,1\}$. If there exist a matrix $K \in \mathbb{R}^{m \times n}$, a positive-definite matrix $P \in \mathbb{R}^{n \times n}$, and scalars $\beta \in(0,1), \varphi \in[1, \infty)$ such that (28), (29) and (30) hold with $\rho \triangleq \frac{p_{0,1}}{p_{0,1}+p_{1,0}}$, then the event-triggered control law (2), (23) guarantees almost sure asymptotic stability of the zero solution $x(t) \equiv 0$ of the closed-loop system dynamics.

Proof: By the ergodic theorem for irreducible Markov chains [51], we have $\lim _{k \rightarrow \infty} \frac{L(k)}{k}=\rho$. Now, since (26) holds, the result follows from Theorem 3.5 .

When we consider transmission attempts at all times by setting $\theta=1$ in 23), Corollary 3.7 recovers a specialization of the result in [40] for linear systems. Furthermore, if we consider $\{l(i) \in\{0,1\}\}_{i \in \mathbb{N}_{0}}$ to be a Bernoulli process, then $\rho$ in Corollary 3.7 is given by $\rho=p$, where $p=p_{0,1}=$ 


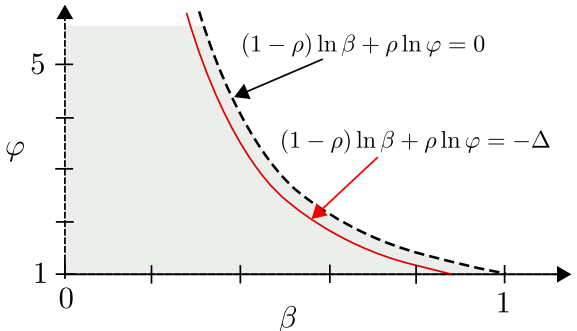

Figure 2. Region for $\beta \in(0,1)$ and $\varphi \in[1, \infty)$ that satisfy [30] for $\rho=0.4$

$p_{1,1}$ denotes the packet loss probability. In this setting, the almost sure stability condition in Corollary 3.7 is tighter than the second-moment stability condition in [36]. Specifically, for this problem setting, the results in [36] can be used to obtain the second-moment stability condition $(1-\rho) \beta+\rho \varphi<$ 1 or equivalently $\ln [(1-\rho) \beta+\rho \varphi]<0$. In comparison to this condition, the stability condition (30) in Corollary 3.7 is tighter. This is because $(1-\rho) \ln \beta+\rho \ln \varphi<\ln [(1-\rho) \beta+\rho \varphi]$ by Jensen's inequality, since $\beta<\varphi$ and $\rho \notin\{0,1\}$.

\section{B. Feedback Gain Design for Event-Triggered Control}

In the following, we outline a numerical method for designing the feedback gain $K \in \mathbb{R}^{m \times n}$, as well as the positivedefinite matrix $P \in \mathbb{R}^{n \times n}$ and the scalar $\beta \in(0,1)$ used in the event-triggered control law (2), (23).

Corollary 3.8: Consider the linear dynamical system (1). Suppose that the process $\{l(i) \in\{0,1\}\}_{i \in \mathbb{N}_{0}}$ characterizing packet exchange failures in the network satisfies (26) with scalar $\rho \in[0,1]$. If there exist a matrix $M \in \mathbb{R}^{m \times n}$, a positivedefinite matrix $Q \in \mathbb{R}^{n \times n}$, and scalars $\beta \in(0,1), \varphi \in[1, \infty)$ such that (30),

$$
\begin{gathered}
{\left[\begin{array}{cc}
\beta Q & (A Q+B M)^{\mathrm{T}} \\
A Q+B M & Q
\end{array}\right] \geq 0,} \\
{\left[\begin{array}{cc}
\varphi Q & (A Q)^{\mathrm{T}} \\
A Q & Q
\end{array}\right] \geq 0,}
\end{gathered}
$$

hold, then the event-triggered control law (2), (23) with $P \triangleq Q^{-1}$ and $K \triangleq M Q^{-1}$ guarantees almost sure asymptotic stability of the zero solution $x(t) \equiv 0$ of the closed-loop system dynamics.

Proof: Using Schur complements (see [52]), we transform (42) and (43), respectively, into

$$
\begin{aligned}
\beta Q-(A Q+B M)^{\mathrm{T}} Q^{-1}(A Q+B M) & \geq 0, \\
\varphi Q-(A Q)^{\mathrm{T}} Q^{-1} A Q & \geq 0 .
\end{aligned}
$$

By multiplying both sides of (44) and (45) from left and right by $Q^{-1}$, we obtain (28) and (29) with $P=Q^{-1}$ and $K=$ $M Q^{-1}$. Thus, the result follows from Theorem 3.5

We remark that the matrix inequalities (42) and (43) are linear in $M \in \mathbb{R}^{m \times n}$ and $Q \in \mathbb{R}^{n \times n}$ for fixed $\beta \in(0,1)$ and $\varphi \in[1, \infty)$. In our method we seek feasible solutions $M$ and $Q$ for linear matrix inequalities (42) and (43) by iterating over a set of values for $\beta \in(0,1)$ and $\varphi \in[1, \infty)$ restricted by the condition (30). It is however noted that we do not need to search $\beta$ and $\varphi$ in the entire range characterized by (30). It turns out to be sufficient to check for larger values of $\beta$ and $\varphi$ that are close to the boundary of the range identified by $(1-\rho) \ln \beta+\rho \ln \varphi=0$. Specifically, we set $\Delta>0$ as a small positive real number, and then we iterate over a set of values for $\beta$ in the range $\left(0, e^{-\frac{\Delta}{1-\rho}}\right]$ to look for feasible solutions $M$ and $Q$ for the linear matrix inequalities (42) and (43) with $\varphi=e^{-\frac{(1-\rho) \ln \beta+\Delta}{\rho}}$. In this approach, we use only $\beta \in(0,1)$, $\varphi \in[1, \infty)$ that are on the curve $(1-\rho) \ln \beta+\rho \ln \varphi=-\Delta$. We illustrate this curve with the solid red line in Fig. 2, where the shaded region corresponds to $\beta$ and $\varphi$ that satisfy (30). Note that picking smaller values for $\Delta>0$ moves the curve towards the boundary. Also, there is no conservatism in not considering $\beta$ and $\varphi$ such that $(1-\rho) \ln \beta+\rho \ln \varphi<-\Delta$. This is because if there exist $M$ and $Q$ that satisfy (42) and (43) for values $\beta=\tilde{\beta}$ and $\varphi=\tilde{\varphi}$, then the same $M$ and $Q$ satisfy (42) and (43) also for larger values $\beta>\tilde{\beta}$ and $\varphi>\tilde{\varphi}$.

\section{ATtACKeR's Perspective}

In order to design cyber-secure control systems, it is essential to understand the risks in networked operation. In this regard, it may be useful to consider the control problem from the perspective of an attacker. An attacker knowledgeable about the networked control system may generate an attack strategy that causes sufficiently frequent packet losses which can result in instability of the closed-loop dynamics. However, the attacker may want to keep the number of attacks as small as possible. One reason in the case of jamming attacks is that monitoring the channel and producing jamming signals consume energy [4]. Moreover, the attacks should be kept minimal to make them less detectable by the system operators.

In this section, we address the question of finding conditions under which the state diverges almost surely (i.e., $\left.\mathbb{P}\left[\lim _{t \rightarrow \infty}\|x(t)\|=\infty\right]=1\right)$. For the discussions and results presented in this section, we consider the case where the plant and the controller attempt to exchange packets at all time instants, that is, $\tau_{i}=i, i \in \mathbb{N}_{0}$. In the event-triggered scheme, this corresponds to the case with $\theta=1$ in (23).

First, we obtain a lower-bound for the long run average number of packet exchange failures by utilizing a characterization that is complementary to (4) in Assumption 2.1

Lemma 4.1: If there exists a scalar $\sigma \in[0,1]$ such that

$$
\sum_{k=1}^{\infty} \mathbb{P}[L(k)<\sigma k]<\infty
$$

where $L(k) \triangleq \sum_{i=0}^{k-1} l(t), k \in \mathbb{N}$, then

$$
\liminf _{k \rightarrow \infty} \frac{L(k)}{k} \geq \sigma
$$

almost surely.

Proof: Using (46), we obtain $\sum_{k=1}^{\infty} \mathbb{P}\left[\sum_{t=0}^{k-1}(1-l(t))>\right.$ $(1-\sigma) k]<\infty$, and hence, by Borel-Cantelli lemma (see [46]),

$$
\limsup _{k \rightarrow \infty} \frac{1}{k} \sum_{t=0}^{k-1}(1-l(t)) \leq 1-\sigma,
$$

almost surely. Noting that $\limsup _{k \rightarrow \infty} \frac{1}{k} \sum_{t=0}^{k-1}(1-l(t))=$ $1-\liminf _{k \rightarrow \infty} \frac{1}{k} \sum_{t=0}^{k-1} l(t)$, we obtain (47) from (48).

The inequality (46) can be considered as a complementary characterization to (4) in Assumption 2.1. Observe that by 
Lemma 3.2 $\rho \in[0,1]$ in (4) characterizes an upper-bound on the long run average number of packet exchange failures. In comparison, as implied by (47), the scalar $\sigma \in[0,1]$ in (46) provides a lower-bound on the long run average number of packet exchange failures.

Notice that a large $\sigma \in[0,1]$ in (47) indicates that due to random losses and malicious attacks, packet exchange failures happen statistically frequently. In such cases, the overall dynamics may become unstable. As mentioned earlier, since malicious attacks often consume energy, the attacker would want to disrupt normal operation and cause unstable behavior with a fewer number of attacks. In the case of jamming attacks, recent works considered game-theoretic methods to investigate the optimal strategy of an attacker when the jamming energy is a constraint in the problem [13] and when it is part of the attacker's cost function [12], [53]. The results obtained there are not directly applicable here, as we investigate sufficient attack rates that cause divergence of the state rather than finding optimal attack strategies.

Our next result indicates how frequently the attacker should cause packet exchange failures to induce instability.

Theorem 4.2: Consider the linear networked control system (11), (2) where packet exchanges between the plant and the controller are attempted at all time instants. Suppose that the process $\{l(t) \in\{0,1\}\}_{t \in \mathbb{N}_{0}}$ characterizing packet exchange failures in the network satisfies (47) with $\sigma \in[0,1]$. If there exist a positive-definite matrix $\hat{P} \in \mathbb{R}^{n \times n}$ and scalars $\hat{\beta} \in$ $(0,1), \hat{\varphi} \in[1, \infty)$ such that

$$
\begin{aligned}
& (A+B K)^{\mathrm{T}} \hat{P}(A+B K)-\hat{\beta} \hat{P} \geq 0, \\
& A^{\mathrm{T}} \hat{P} A-\hat{\varphi} \hat{P} \geq 0 \\
& (1-\sigma) \ln \hat{\beta}+\sigma \ln \hat{\varphi}>0
\end{aligned}
$$

then $\lim _{t \rightarrow \infty}\|x(t)\|=\infty$, almost surely.

Proof: Consider the Lyapunov-like function $V(\cdot)$ given by $V(x) \triangleq x^{\mathrm{T}} \hat{P} x, x \in \mathbb{R}^{n}$. For the case $\tau_{i}=i, i \in \mathbb{N}_{0}$, by (1), (2), we have

$$
\begin{aligned}
V(x(t+1))= & x^{\mathrm{T}}(t)(A+(1-l(t)) B K)^{\mathrm{T}} \hat{P} \\
& \cdot(A+(1-l(t)) B K) x(t), t \in \mathbb{N}_{0} .
\end{aligned}
$$

From (49), (50), and (52), this can be bounded by

$$
V(x(t+1)) \geq(1-l(t)) \hat{\beta} V(x(t))+l(t) \hat{\varphi} V(x(t))
$$

for $t \in \mathbb{N}_{0}$. Now, let $\eta(k) \triangleq \prod_{t=0}^{k-1}((1-l(t)) \hat{\beta}+l(t) \hat{\varphi})$. It follows from (53) that

$$
V(x(k)) \geq \eta(k) V(x(0))
$$

for $k \in \mathbb{N}$. Furthermore, since $\ln [(1-q) \hat{\beta}+q \hat{\varphi}]=(1-$ q) $\ln \hat{\beta}+q \ln \hat{\varphi}$ for $q \in\{0,1\}$, we have

$$
\ln \eta(k)=\sum_{t=0}^{k-1}(1-l(t)) \ln \hat{\beta}+\sum_{t=0}^{k-1} l(t) \ln \hat{\varphi}
$$

Now since $\hat{\beta} \in(0,1)$, we have $\ln \hat{\beta}<0$, and hence by (47),

$$
\begin{aligned}
& \liminf _{k \rightarrow \infty} \frac{1}{k} \sum_{t=0}^{k-1}(1-l(t)) \ln \hat{\beta}=(\ln \hat{\beta}) \limsup _{k \rightarrow \infty} \frac{1}{k} \sum_{t=0}^{k-1}(1-l(t)) \\
& =(\ln \hat{\beta})\left(1-\liminf _{k \rightarrow \infty} \frac{1}{k} \sum_{t=0}^{k-1} l(t)\right) \geq(\ln \hat{\beta})(1-\sigma) .
\end{aligned}
$$

Furthermore, since $\ln \hat{\varphi} \geq 0$, it follows from (47) that $\lim \inf _{k \rightarrow \infty} \frac{1}{k} \sum_{t=0}^{k-1} l(t) \ln \hat{\varphi} \geq \sigma \ln \hat{\varphi}$. Consequently, by (51),

$$
\begin{aligned}
& \liminf _{k \rightarrow \infty} \frac{\ln \eta(k)}{k} \geq \liminf _{k \rightarrow \infty} \frac{1}{k} \sum_{t=0}^{k-1}(1-l(t)) \ln \hat{\beta} \\
& \quad+\liminf _{k \rightarrow \infty} \frac{1}{k} \sum_{t=0}^{k-1} l(t) \ln \hat{\varphi} \geq(1-\sigma) \ln \hat{\beta}+\sigma \ln \hat{\varphi}>0,
\end{aligned}
$$

almost surely. As a consequence, $\lim _{k \rightarrow \infty} \ln \eta(k)=\infty$, and hence, $\lim _{k \rightarrow \infty} \eta(k)=\infty$, almost surely. Thus, it follows from (54) that $\mathbb{P}\left[\lim _{t \rightarrow \infty} V(x(t))=\infty\right]=1$, which implies that $\lim _{t \rightarrow \infty}\|x(t)\|=\infty$, almost surely.

Theorem 4.2 provides sufficient conditions (49)-(51) to assess instability of the closed-loop system (1), (2). These conditions are complementary to the stability conditions (28) (30) in Theorem 3.5 This point is further illustrated by focusing on the scalar systems case.

Example 4.3: Consider the scalar system (1) with $A, B \in \mathbb{R}$. Then, conditions (49), (50) as well as (28), (29) can be satisfied by $\hat{P}=P=1, \hat{\beta}=\beta=(A+B K)^{2}$, and $\hat{\varphi}=\varphi=A^{2}$. Now, if $\lim _{k \rightarrow \infty} \frac{L(k)}{k}$ exists and is a fixed constant, we can set $\sigma=\rho=\lim _{k \rightarrow \infty} \frac{L(k)}{k}$ in (30) and (51) to obtain the stability condition

$$
\left(1-\lim _{k \rightarrow \infty} \frac{L(k)}{k}\right)(A+B K)^{2}+\lim _{k \rightarrow \infty} \frac{L(k)}{k} A^{2}<0,
$$

and the instability condition

$$
\left(1-\lim _{k \rightarrow \infty} \frac{L(k)}{k}\right)(A+B K)^{2}+\lim _{k \rightarrow \infty} \frac{L(k)}{k} A^{2}>0 .
$$

The limit $\lim _{k \rightarrow \infty} \frac{L(k)}{k}$ is a fixed constant for example when the packet losses are Bernoulli-type or periodic. In those cases, (55) and (56) indicate that Theorems 3.5 and 4.2 provide tight stability/instability conditions for scalar systems. On the other hand, for multi-dimensional systems, scalars $\beta$ and $\hat{\beta}$ as well as $\varphi$ and $\hat{\varphi}$ may not always be selected equal to obtain tight results. Furthermore, under random packet losses and malicious attacks, $\lim _{k \rightarrow \infty} \frac{L(k)}{k}$ may not always exist and hence there may be a discrepancy between $\rho$ and $\sigma$ in (26) and (47).

Proposition 4.4 below provides a range of values for $\sigma$ that satisfy (46) in the case where the network faces random and malicious packet losses.

Proposition 4.4: Consider the packet exchange failure indicator process $\{l(t) \in\{0,1\}\}_{t \in \mathbb{N}_{0}}$ given by (13) where $\left\{l_{\mathrm{R}}(t) \in\{0,1\}\right\}_{t \in \mathbb{N}_{0}}$ and $\left\{l_{\mathrm{M}}(t) \in\{0,1\}\right\}_{t \in \mathbb{N}_{0}}$ are mutually independent. Suppose there exists $\sigma_{\mathrm{M}} \in(0,1)$ such that

$$
\sum_{k=1}^{\infty} \mathbb{P}\left[\sum_{t=0}^{k-1} l_{\mathrm{M}}(t)<\sigma_{\mathrm{M}} k\right]<\infty .
$$


Furthermore, suppose $\left\{l_{\mathrm{R}}(t) \in\{0,1\}\right\}_{t \in \mathbb{N}_{0}}$ satisfies (7) with $p_{0} \in(0,1)$. Then (46) holds for all $\sigma \in\left(0,1-p_{0}\left(1-\sigma_{\mathrm{M}}\right)\right)$.

Proof: First, by using (13), we obtain

$$
\begin{aligned}
& \mathbb{P}\left[\sum_{t=0}^{k-1} l(t)<\sigma k\right]=\mathbb{P}\left[\sum_{t=0}^{k-1}(1-l(t))>(1-\sigma) k\right] \\
& \quad=\mathbb{P}\left[\sum_{t=0}^{k-1}\left(1-l_{\mathrm{R}}(t)\right)\left(1-l_{\mathrm{M}}(t)\right)>(1-\sigma) k\right], k \in \mathbb{N} .
\end{aligned}
$$

Furthermore, it follows from (57) that $\sum_{k=1}^{\infty} \mathbb{P}\left[\sum_{t=0}^{k-1}(1-\right.$ $\left.\left.l_{\mathrm{M}}(t)\right)>\left(1-\sigma_{\mathrm{M}}\right) k\right]=\sum_{k=1}^{\infty} \mathbb{P}\left[\sum_{t=0}^{k-1} l_{\mathrm{M}}(i)<\sigma_{\mathrm{M}} k\right]<\infty$. Hence, $\{\chi(i) \triangleq\{0,1\}\}_{i \in \mathbb{N}_{0}}$ defined by $\chi(i)=1-l_{\mathrm{M}}(i), i \in$ $\mathbb{N}_{0}$, satisfies 68 with $\tilde{w}=1-\sigma_{\mathrm{M}}<1$. Furthermore, $\{\xi(i) \triangleq\{0,1\}\}_{i \in \mathbb{N}_{0}}$ defined by $\xi(i)=1-l_{\mathrm{R}}(i), i \in \mathbb{N}_{0}$, satisfies (67) with $\tilde{p}=p_{0} \in(0,1)$. We then have from Lemma A.1 that

$$
\sum_{k=1}^{\infty} \mathbb{P}\left[\sum_{t=0}^{k-1}\left(1-l_{\mathrm{R}}(t)\right)\left(1-l_{\mathrm{M}}(t)\right)>\varrho k\right]<\infty
$$

for all $\varrho \in\left(p_{0}\left(1-\sigma_{\mathrm{M}}\right), 1-\sigma_{\mathrm{M}}\right)$.

In the rest of the proof, we will show that (59) holds also for $\varrho \in\left[1-\sigma_{\mathrm{M}}, 1\right)$. To this end, let $\varrho^{\prime} \triangleq \frac{p_{0}\left(1-\sigma_{\mathrm{M}}\right)+1-\sigma_{\mathrm{M}}}{2}$. Since $\varrho^{\prime} \in\left(p_{0}\left(1-\sigma_{\mathrm{M}}\right), 1-\sigma_{\mathrm{M}}\right)$, by (59), we get $\lambda \triangleq$ $\sum_{k=1}^{\infty} \mathbb{P}\left[\sum_{t=0}^{k-1}\left(1-l_{\mathrm{R}}(t)\right)\left(1-l_{\mathrm{M}}(t)\right)>\varrho^{\prime} k\right]<\infty$. Furthermore, for all $\varrho \in\left[1-\sigma_{\mathrm{M}}, 1\right)$ we have $\varrho \geq \varrho^{\prime}$ and hence

$$
\begin{aligned}
& \mathbb{P}\left[\sum_{t=0}^{k-1}\left(1-l_{\mathrm{R}}(t)\right)\left(1-l_{\mathrm{M}}(t)\right)>\varrho k\right] \\
& \quad \leq \mathbb{P}\left[\sum_{t=0}^{k-1}\left(1-l_{\mathrm{R}}(t)\right)\left(1-l_{\mathrm{M}}(t)\right)>\varrho^{\prime} k\right], \quad k \in \mathbb{N} .
\end{aligned}
$$

Thus, for $\varrho \in\left[1-\sigma_{\mathrm{M}}, 1\right), \sum_{k=1}^{\infty} \mathbb{P}\left[\sum_{t=0}^{k-1}\left(1-l_{\mathrm{R}}(t)\right)(1-\right.$ $\left.\left.l_{\mathrm{M}}(t)\right)>\varrho k\right] \leq \lambda<\infty$. Therefore, (59) holds for all $\varrho \in$ $\left(p_{0}\left(1-\sigma_{\mathrm{M}}\right), 1\right)=\left(p_{0}\left(1-\sigma_{\mathrm{M}}\right), 1-\sigma_{\mathrm{M}}\right) \cup\left[1-\sigma_{\mathrm{M}}, 1\right)$. Now since $\sigma=1-\varrho$, it follows from (58) that (46) holds for all $\sigma \in\left(0,1-p_{0}\left(1-\sigma_{\mathrm{M}}\right)\right)$.

Proposition 4.4 shows that when malicious attacks are independent of the random losses and they satisfy (57), the inequalities (46) and (47) (due to Lemma 4.1) hold for a range of values of $\sigma$. This result indicates the effects of independent random packet losses and malicious attacks on the asymptotic ratio of packet exchange attempt failures over all attempts.

The next result is concerned with the scenarios where random packet losses and malicious attacks need not be independent.

Proposition 4.5: Consider the packet exchange failure indicator process $\{l(t) \in\{0,1\}\}_{t \in \mathbb{N}_{0}}$ given by (13). Suppose there exists $\sigma_{\mathrm{M}} \in(0,1)$ such that (57) holds. Furthermore, suppose $\left\{l_{\mathrm{R}}(t) \in\{0,1\}\right\}_{t \in \mathbb{N}_{0}}$ satisfies (7) with $p_{0} \in(0,1)$. Then (46) holds for all $\sigma \in\left(0, \max \left\{1-p_{0}, \sigma_{\mathrm{M}}\right\}\right)$.

Proof: We will show that (46) holds for the cases: 1) $\max \left\{1-p_{0}, \sigma_{\mathrm{M}}\right\}=1-p_{0}$ and 2) $\max \left\{1-p_{0}, \sigma_{\mathrm{M}}\right\}=\sigma_{\mathrm{M}}$.
First, if $\max \left\{1-p_{0}, \sigma_{\mathrm{M}}\right\}=1-p_{0}$, then noting that $\sum_{t=0}^{k-1} l_{\mathrm{R}}(t) \leq \sum_{t=0}^{k-1} l(t)$, we obtain

$$
\begin{aligned}
\mathbb{P}\left[\sum_{t=0}^{k-1} l(t)<\sigma k\right] & =\mathbb{P}\left[\sum_{t=0}^{k-1}(1-l(t))>(1-\sigma) k\right] \\
& \leq \mathbb{P}\left[\sum_{t=0}^{k-1}\left(1-l_{\mathrm{R}}(t)\right)>(1-\sigma) k\right],
\end{aligned}
$$

for $k \in \mathbb{N}$. Now, $\{\chi(i) \triangleq\{0,1\}\}_{i \in \mathbb{N}_{0}}$ with $\chi(i)=1, i \in \mathbb{N}_{0}$, satisfies (68) with $\tilde{w}=1$. Furthermore, $\{\xi(i) \triangleq\{0,1\}\}_{i \in \mathbb{N}_{0}}$ with $\xi(i)=1-l_{\mathrm{R}}(i), i \in \mathbb{N}_{0}$, satisfies (67) with $\tilde{p}=p_{0} \in$ $(0,1)$. Since $1-\sigma>p_{0}$, we have from Lemma A.1 that $\sum_{k=1}^{\infty} \mathbb{P}\left[\sum_{t=0}^{k-1}\left(1-l_{\mathrm{R}}(t)\right)>(1-\sigma) k\right]<\infty$. Hence, by (60), we have (46).

Next, if $\max \left\{1-p_{0}, \sigma_{\mathrm{M}}\right\}=\sigma_{\mathrm{M}}$, then since $\sum_{t=0}^{k-1} l_{\mathrm{M}}(t) \leq$ $\sum_{t=0}^{k-1} l(t)$ and $\sigma<\sigma_{\mathrm{M}}$, we get

$$
\mathbb{P}\left[\sum_{t=0}^{k-1} l(t)<\sigma k\right] \leq \mathbb{P}\left[\sum_{t=0}^{k-1} l_{\mathrm{M}}(t)<\sigma_{\mathrm{M}} k\right], \quad k \in \mathbb{N} .
$$

Consequently, (57) and (61) imply (46).

Proposition 4.5 provides a range for $\sigma$ in (46) when we consider the case where random packet losses and malicious attacks may be dependent. This range is smaller in comparison to the one provided in Proposition 4.4 for the independent case. This is because Proposition 4.5 deals with scenarios including the worst case from the perspective of the attacker. In that scenario, the malicious attacks and random packet losses happen at the same time instants, and hence, the statistical frequency of the overall packet exchange failures cannot exceed the maximum of the frequencies of malicious attacks and random packet losses. We remark that there are other scenarios where the attacks depend on the random packet losses. For instance, the attacker may intentionally avoid attacking when there is already a random packet loss. This scenario is characterized in the mathematical setting by $l_{\mathrm{R}}(t) l_{\mathrm{M}}(t)=0, t \in \mathbb{N}_{0}$. For this scenario, the following proposition provides a range of $\sigma$ that satisfy (46).

Proposition 4.6: Consider the packet exchange failure indicator process $\{l(t) \in\{0,1\}\}_{t \in \mathbb{N}_{0}}$ given by (13). Suppose $\left\{l_{\mathrm{R}}(t) \in\{0,1\}\right\}_{t \in \mathbb{N}_{0}}$ satisfies (7) with $p_{0} \in(0,1)$. Furthermore, suppose $l_{\mathrm{R}}(t) l_{\mathrm{M}}(t)=0, t \in \mathbb{N}_{0}$, and there exists $\sigma_{\mathrm{M}} \in(0,1)$ such that (57) holds. If $1-p_{0}+\sigma_{\mathrm{M}} \leq 1$, then (46) holds for all $\sigma \in\left(0,1-p_{0}+\sigma_{\mathrm{M}}\right)$.

Proof: First let $\epsilon \triangleq 1-p_{0}+\sigma_{\mathrm{M}}-\sigma$, and define $\sigma_{1} \triangleq$ $\max \left\{0,1-p_{0}-\frac{\epsilon}{2}\right\}, \sigma_{2} \triangleq \max \left\{0, \sigma_{\mathrm{M}}-\frac{\epsilon}{2}\right\}$. Note that $\sigma_{1}+\sigma_{2} \geq$ $\sigma$. Now since $l_{\mathrm{R}}(t) l_{\mathrm{M}}(t)=0, t \in \mathbb{N}_{0}$, we have from (13) that $\sum_{t=0}^{k-1} l(t)=\sum_{t=0}^{k-1} l_{\mathrm{R}}(t)+\sum_{t=0}^{k-1} l_{\mathrm{M}}(t)$. As a result

$$
\begin{aligned}
& \mathbb{P}\left[\sum_{t=0}^{k-1} l(t)<\sigma k\right]=\mathbb{P}\left[\sum_{t=0}^{k-1} l_{\mathrm{R}}(t)+\sum_{t=0}^{k-1} l_{\mathrm{M}}(t)<\sigma k\right] \\
& \leq \mathbb{P}\left[\sum_{t=0}^{k-1} l_{\mathrm{R}}(t)+\sum_{t=0}^{k-1} l_{\mathrm{M}}(t)<\sigma_{1} k+\sigma_{2} k\right] \\
& \leq \mathbb{P}\left[\sum_{t=0}^{k-1} l_{\mathrm{R}}(t)<\sigma_{1} k\right]+\mathbb{P}\left[\sum_{t=0}^{k-1} l_{\mathrm{M}}(t)<\sigma_{2} k\right], k \in \mathbb{N} .
\end{aligned}
$$


If $\sigma_{1}=0$, then $\mathbb{P}\left[\sum_{t=0}^{k-1} l_{\mathrm{R}}(t)<\sigma_{1} k\right]=0$, and hence $\sum_{k=1}^{\infty} \mathbb{P}\left[\sum_{t=0}^{k-1} l_{\mathrm{R}}(t)<\sigma_{1} k\right]=0<\infty$. If, on the other hand, $\sigma_{1}>0$, then we can utilize Lemma A.1. Specifically, $\{\chi(i) \triangleq\{0,1\}\}_{i \in \mathbb{N}_{0}}$ with $\chi(i)=1, i \in \mathbb{N}_{0}$, satisfies (68) with $\tilde{w}=1$. Furthermore, $\{\xi(i) \triangleq\{0,1\}\}_{i \in \mathbb{N}_{0}}$ with $\xi(i)=1-l_{\mathrm{R}}(i), i \in \mathbb{N}_{0}$, satisfies (67) with $\tilde{p}=p_{0} \in(0,1)$. Since $\sigma_{1}>0$, it means that $\sigma_{1}=1-p_{0}-\frac{\epsilon}{2}$. Now, since $\epsilon>0$, we have $\left(1-\sigma_{1}\right) \in\left(p_{0}, 1\right)$. Consequently, we obtain from Lemma A.1 that $\sum_{k=1}^{\infty} \mathbb{P}\left[\sum_{t=0}^{k-1}\left(1-l_{\mathrm{R}}(t)\right)>\left(1-\sigma_{1}\right) k\right]<\infty$, and hence,

$$
\begin{aligned}
\sum_{k=1}^{\infty} & \mathbb{P}\left[\sum_{t=0}^{k-1} l_{\mathrm{R}}(t)<\sigma_{1} k\right] \\
\quad= & \sum_{k=1}^{\infty} \mathbb{P}\left[\sum_{t=0}^{k-1}\left(1-l_{\mathrm{R}}(t)\right)>\left(1-\sigma_{1}\right) k\right]<\infty .
\end{aligned}
$$

Similarly, if $\sigma_{2}=0$, then $\mathbb{P}\left[\sum_{t=0}^{k-1} l_{\mathrm{M}}(t)<\sigma_{2} k\right]=0$, and hence $\sum_{k=1}^{\infty} \mathbb{P}\left[\sum_{t=0}^{k-1} l_{\mathrm{M}}(t)<\sigma_{2} k\right]=0<\infty$. On the other hand, if $\sigma_{2}=\sigma_{\mathrm{M}}-\frac{\epsilon}{2}>0$, since $\epsilon>0$, we have $\sigma_{2}<\sigma_{\mathrm{M}}$. Thus, $\mathbb{P}\left[\sum_{t=0}^{k-1} l_{\mathrm{M}}(t)<\sigma_{2} k\right] \leq \mathbb{P}\left[\sum_{t=0}^{k-1} l_{\mathrm{M}}(t)<\sigma_{\mathrm{M}} k\right]$. It then follows from (57) that

$$
\sum_{k=1}^{\infty} \mathbb{P}\left[\sum_{t=0}^{k-1} l_{\mathrm{M}}(t)<\sigma_{2} k\right] \leq \sum_{k=1}^{\infty} \mathbb{P}\left[\sum_{t=0}^{k-1} l_{\mathrm{M}}(t)<\sigma_{\mathrm{M}} k\right]<\infty .
$$

Finally, (46) follows from (62)-64).

An attacker that is knowledgeable about the random packet losses in the network may avoid placing malicious attacks when random packet losses occur. Proposition 4.6 provides a range of values of $\sigma$ such that the inequality (46) holds when the attacker follows this strategy. Compared to the case where attacks and random packet losses are independent, this strategy would increase the overall number of packet exchange failures, even though the number of attacks may be the same. The reason is that in the independent case, the attacks and random packet losses may occasionally happen at the same time, reducing the total packet failure count. Noe that the range of $\sigma$ in Proposition 4.6 is larger than that in Proposition 4.4 where the attacks and random packet losses are independent, even though in both results the malicious attacks satisfy (57) with the same $\sigma_{\mathrm{M}} \in(0,1)$. In Section $\nabla-\mathrm{B}$, we discuss and compare two attack strategies independent/dependent on random packet losses. Both strategies cause instability for certain feedback gain and event-triggering mechanism parameters.

It is important to note that particular choices of the controller parameters may result in instability when $\sigma \in[0,1]$ is large. If the packet exchange failures are known to happen statistically frequently, that is, if $\sigma$ is large, then the feedback gain $K$ and the event-triggering mechanism parameters $\beta$ and $P$ should be redesigned to ensure stability. In such cases, Theorem 3.5 and Corollary 3.8 can be employed with $\rho \geq \sigma$ that satisfies (4) or (26).

\section{NUMERICAL EXAMPLES}

In this section we present numerical examples to illustrate our results provided in Sections [I-IV

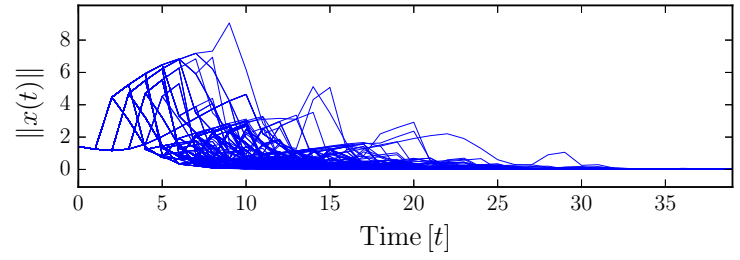

Figure 3. Sample paths of the state norm

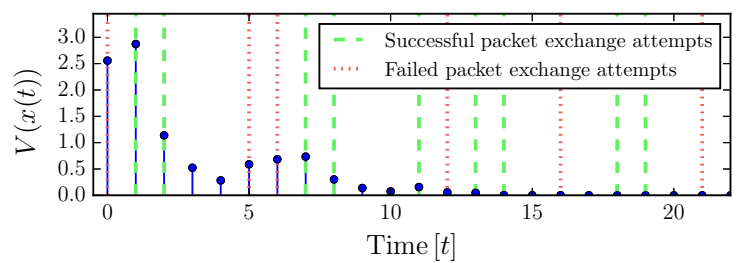

Figure 4. A sample path of Lyapunov-like function $V(\cdot)$

A) Example 1: We consider the system (1) with

$$
A \triangleq\left[\begin{array}{cc}
1 & 0.1 \\
-0.5 & 1.1
\end{array}\right], \quad B \triangleq\left[\begin{array}{l}
0.1 \\
1.2
\end{array}\right]
$$

We use the event-triggering control law (2), (23) for stabilization of (1) over a network that faces independent random packet losses and malicious attacks. Specifically, random packet losses are assumed to be characterized by the Markov chain $\left\{l_{\mathrm{R}}(i) \in\{0,1\}\right\}_{i \in \mathbb{N}_{0}}$ with initial distribution $\vartheta_{0}=0, \vartheta_{1}=1$, and transition probabilities $p_{0,1}(i) \triangleq$ $0.2+0.03 \sin ^{2}(0.1 i), p_{1,1}(i) \triangleq 0.2+0.03 \cos ^{2}(0.1 i)$, and $p_{q, 0}(i)=1-p_{q, 1}(i), q \in\{0,1\}, i \in \mathbb{N}_{0}$. Note that $\left\{l_{\mathrm{R}}(i) \in\{0,1\}\right\}_{i \in \mathbb{N}_{0}}$ satisfies (6) and (7) with $p_{1}=0.23$ and $p_{0}=0.8$. Furthermore, the network is subject to jamming attacks that is independent of $\left\{l_{\mathrm{R}}(i) \in\{0,1\}\right\}_{i \in \mathbb{N}_{0}}$ and satisfies (9) with $\kappa=2$ and $\tau=5$. By Lemma 2.3, (11) holds with $\rho_{\mathrm{M}}=0.21$ since $\rho_{\mathrm{M}}=0.21>\frac{1}{\tau}=0.2$. Furthermore, note that $p_{1}+p_{0} \rho_{\mathrm{M}}<0.4$. Hence, it follows from Proposition 2.4 that for $\rho=0.4$, (4) of Assumption 2.1 holds, which implies (26) through Lemma 3.2 .

We designed the controller based on the procedure in Section III-B and obtained the matrices

$$
Q=\left[\begin{array}{cc}
0.618 & -2.119 \\
-2.119 & 28.214
\end{array}\right], M=\left[\begin{array}{ll}
0.202 & -20.405
\end{array}\right],
$$

and scalars $\beta=0.55, \varphi=2.4516$ satisfy (42), (43), and (30) with $\rho=0.4$. Hence, it follows from Corollary 3.8 that the event-triggered control law (2), (23) with $P=Q^{-1}$ and $K=M Q^{-1}$ guarantees almost sure asymptotic stabilization.

We generated 250 sample state trajectories using the same initial condition $x_{0}=[1,1]^{\mathrm{T}}$ and the event-triggering mechanism parameter $\theta=1000$, but with different sample paths for $l_{\mathrm{R}}(\cdot)$ and $l_{\mathrm{M}}(\cdot)$. We can check in Fig. 3 that all state trajectories go to the origin. The same is true for the Lyapunovlike function $V(x(t))$. We show a single sample trajectory of $V(\cdot)$ in Fig. 4 The Lyapunov-like function $V(\cdot)$ converges to zero, but notice that it is not monotonically decreasing. The Lyapunov-like function $V(\cdot)$ increases in two situations. First, when packet exchange attempts fail, $V(\cdot)$ may grow and take a larger value at the next packet exchange attempt instant due to unstable dynamics of the uncontrolled system. Second, $V(\cdot)$ may also increase some time after a successful packet 


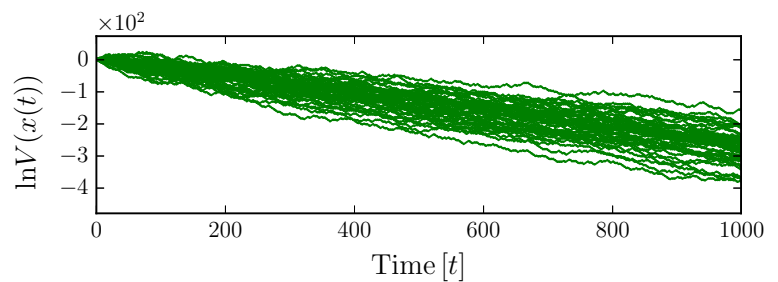

Figure 5. Sample paths of $\ln V(x(\cdot))$ under malicious attack 65 with $\tau=3$

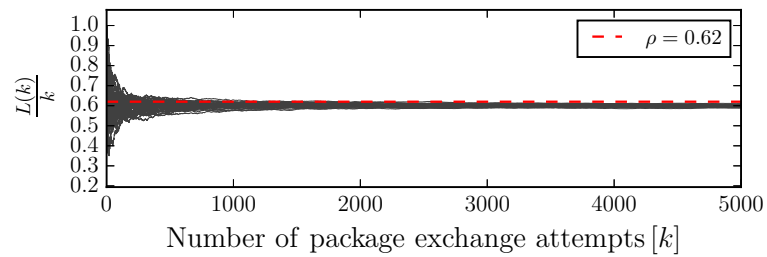

Figure 6. Sample paths of the average number of packet exchange attempt failures $(L(k) / k)$ under malicious attack 65 with $\tau=3$

exchange between the plant and the controller. This is because the constant control input updated with the packet exchange becomes ineffective after some time. Note that eventually a new packet exchange attempt is triggered before $V(\cdot)$ leaves the bound identified in the event-triggering condition (23).

B) Example 2: Our goal in this example is to illustrate effects of different attack strategies discussed in Section IV Here, we consider a scalar linear system (1) with $A=2$ and $B=1$. Its initial state is set to $x_{0}=1$. Furthermore, the feedback gain and the event-triggering mechanism parameters in (2) and (23) are given by $K=-1.75, \beta=0.0625, P=1$. We set the packet exchange events to be triggered at all time instants. This is done with $\theta=1$ in (23).

The random packet losses in the network are characterized by the Markov chain $\left\{l_{\mathrm{R}}(t) \in\{0,1\}\right\}_{t \in \mathbb{N}_{0}}$ with initial distribution $\vartheta_{0}=0, \vartheta_{1}=1$, and transition probabilities $p_{0,1}(t) \triangleq 0.4+0.01 \cos (0.1 t), p_{1,1}(t) \triangleq 0.4+0.01 \sin (0.1 t)$, and $p_{q, 0}(t)=1-p_{q, 1}(t), q \in\{0,1\}, t \in \mathbb{N}_{0}$. Note that $l_{\mathrm{R}}(\cdot)$ satisfies (6) and (7) with $p_{1}=0.41$ and $p_{0}=0.61$.

We consider two attack strategies described by (9) and discuss stability properties of the closed-loop system.

(i) Random-Loss-Independent Attack Strategy: We consider the strategy given by $l_{\mathrm{M}}(0) \triangleq 0$, and

$$
l_{\mathrm{M}}(t) \triangleq \begin{cases}1, & \text { if } \sum_{i=0}^{t-1} l_{\mathrm{M}}(i) \leq \frac{t+1}{\tau}-1, \quad t \in \mathbb{N} . \\ 0, & \text { otherwise, }\end{cases}
$$

Note that (65) satisfies (9) with $\kappa=0$. In this strategy, the attacker uses the total count of all attacks prior to time $t$ to check whether placing an attack at time $t$ would meet the requirement in (9) or not. The attacker causes a packet exchange failure at time $t$ if (9) still holds at time $t$ (i.e., $\sum_{i=0}^{t} l_{\mathrm{M}}(i) \leq \frac{t+1}{\tau}$ ). Under this strategy, attacks are independent of random packet losses and the attack times become periodic with period $\tau$ when $\tau$ is an integer. We will assess stability/instability of the closed-loop system with two different values of $\tau$.

First, we consider $\tau=3$, that is, the attacker prevents packet exchanges once in every 3 steps. In this case the closed-loop system is stable despite the attack. We use Theorem 3.5 to show stability as follows. By Lemma 2.3, (11) holds with $\rho_{\mathrm{M}}=0.3334>\frac{1}{\tau}=\frac{1}{3}$. Now, note that $p_{1}+p_{0} \rho_{\mathrm{M}}<0.62$.

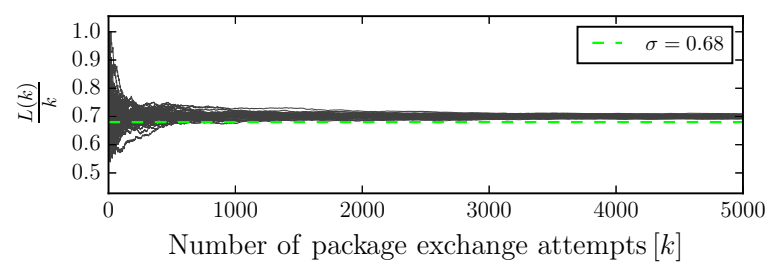

Figure 7. Sample paths of the average number of packet exchange attempt failures $(L(k) / k)$ under malicious attack with $\tau=2$

Since $l_{\mathrm{R}}(\cdot)$ and $l_{\mathrm{M}}(\cdot)$ are independent, it follows from Proposition 2.4 that (4) in Assumption 2.1 holds for $\rho=0.62$, which implies (26) through Lemma 3.2 Further, (28)-(30) hold with $P=1, \beta=0.0625$, and $\varphi=4$. By Theorem 3.5, the eventtriggered control law (2), (23) with $P=1, \beta=0.0625$, and $K=-1.75$ guarantees almost sure asymptotic stabilization.

Fig. 5 shows 50 sample trajectories of $\ln V(x(\cdot))$ where $V(x(t)) \triangleq x^{2}(t)$. These trajectories are obtained under malicious attack (65) but with different sample paths for $\left\{l_{\mathrm{R}}(t) \in\right.$ $\{0,1\}\}_{t \in \mathbb{N}_{0}}$. Note that all trajectories of $\ln V(x(\cdot))$ approach $-\infty$, indicating convergence of the state to 0 . Moreover, in Fig. 6, we show sample trajectories of the average number of packet exchange attempt failures. Observe that the long run average number of packet failures is small enough to guarantee stability $\left(\lim \sup _{k \rightarrow \infty} \frac{L(k)}{k} \leq \rho=0.62\right)$.

Next, we consider 65) with $\tau=2$, i.e., the malicious attacker prevents every other packet exchange attempt. With $\tau=2$, the closed-loop system becomes unstable. We can show this through Theorem 4.2 as follows. First, note that in this case, (65) implies (57) with $\sigma_{\mathrm{M}} \triangleq 0.49$. To see this, we observe that $\sum_{t=0}^{k-1} l_{\mathrm{M}}(t) \geq \frac{k}{\tau}-1=\frac{k}{2}-1$ for all $k \in \mathbb{N}$. Next, using Markov's inequality we obtain

$$
\begin{aligned}
& \mathbb{P}\left[\sum_{t=0}^{k-1} l_{\mathrm{M}}(t)<\sigma_{\mathrm{M}} k\right]=\mathbb{P}\left[\sum_{t=0}^{k-1}\left(1-l_{\mathrm{M}}(t)\right)>\left(1-\sigma_{\mathrm{M}}\right) k\right] \\
& \quad \leq \mathbb{P}\left[e^{\sum_{t=0}^{k-1}\left(1-l_{\mathrm{M}}(t)\right)} \geq e^{\left(1-\sigma_{\mathrm{M}}\right) k}\right] \\
& \quad \leq e^{-\left(1-\sigma_{\mathrm{M}}\right) k} \mathbb{E}\left[e^{\sum_{t=0}^{k-1}\left(1-l_{\mathrm{M}}(t)\right)}\right] \leq e^{-\left(1-\sigma_{\mathrm{M}}\right) k} e^{1+\frac{k}{2}}
\end{aligned}
$$

for $k \in \mathbb{N}$. Consequently, since $\sigma_{\mathrm{M}}<\frac{1}{\tau}=\frac{1}{2}$, we have $\sum_{k=1}^{\infty} \mathbb{P}\left[\sum_{t=0}^{k-1} l_{\mathrm{M}}(t)<\sigma_{\mathrm{M}} k\right] \leq \sum_{k=1}^{\infty} e^{-\left(1-\sigma_{\mathrm{M}}\right) k} e^{1+\frac{k}{2}}=$ $e^{\frac{1}{2}+\sigma_{\mathrm{M}}}\left(1-e^{\sigma_{\mathrm{M}}-\frac{1}{2}}\right)^{-1}<\infty$, which implies (57). Now, note that $1-p_{0}\left(1-\sigma_{\mathrm{M}}\right)>0.68$. Hence, by Proposition 4.4 we have (46) for $\sigma=0.68$. Consequently, by Lemma 4.1, (47) holds for $\sigma=0.68$. Furthermore, inequalities (49)-(51) hold with $\hat{P}=1, \hat{\beta}=0.0625$, and $\hat{\varphi}=4$. It follows from Theorem 4.2 that the closed-loop system with $K=-1.75$ is unstable.

This example shows that an attacker can destabilize the system by reducing $\tau$ from 3 to 2 and hence causing higher number of packet losses on average. As illustrated in Fig. 7 , when $\tau=2$, in the long run, the average number of packet failures becomes larger $\left(\liminf _{k \rightarrow \infty} \frac{L(k)}{k} \geq \sigma=0.68\right)$ compared to the case with $\tau=3$ in Fig. 6

(ii) Selective Attack Strategy: Next, we consider the case where the attacker is knowledgeable about the random packet losses in the network. To describe this strategy we let

$l_{\mathrm{M}}(t) \triangleq \begin{cases}1, & \text { if } l_{\mathrm{R}}(t)=0 \text { and } \sum_{i=0}^{t-1} l_{\mathrm{M}}(i) \leq \frac{t+1}{\tau}-1, \\ 0, & \text { otherwise }\end{cases}$ 


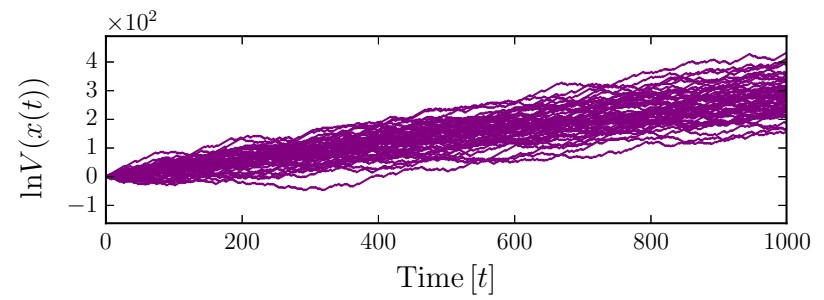

Figure 8. Sample paths of $\ln V(x(\cdot))$ under malicious attack with $\tau=3$

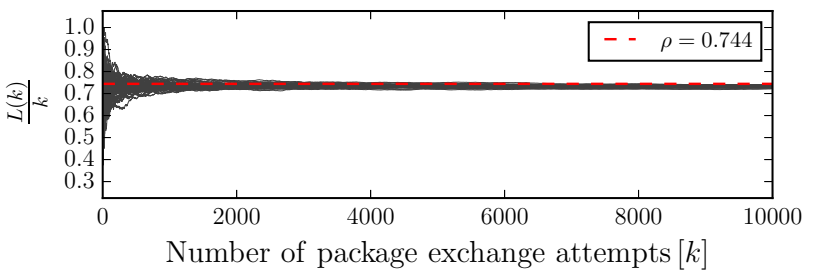

Figure 9. Sample paths of the average number of packet exchange attempt failures $(L(k) / k)$ under malicious attack (66) with $\tau=3$

for $t \in \mathbb{N}$ and $l_{\mathrm{M}}(0)=0$. This strategy is similar to the one given by (65) in that it satisfies (9) with $i=t$ and $\kappa=0$. However, an attacker following (66) utilizes random packet loss information at time $t$, by not placing an attack when $l_{\mathrm{R}}(t)=1$ (indicating packet failures due to random errors).

To compare, we set $\tau=3$, under which the first strategy (65) cannot destabilize the system. From the simulations, we notice that with $\tau=3$, the selective attack strategy causes the system state to diverge (see Fig. 8 for 50 sample paths of $\ln V(x(t))$ with $\left.V(x(t)) \triangleq x^{2}(t)\right)$. In fact, we observe from Fig. 9 that (47) holds with $\sigma=0.7$. This $\sigma$ satisfies condition (51) of Theorem 4.2, indicating instability.

Next, we consider an extension to the attack model in (66). In this model, the attacker places attacks whenever $l_{\mathrm{R}}(t)=$ $0, \sum_{i=0}^{t-1} l_{\mathrm{M}}(i) \leq \frac{t+1}{\tau}-1$, and $\ln V(x(t)) \leq \zeta$. From the simulations with $\zeta=50$ and $\tau=3$, we see that the state does not diverge, but the attacker is able to keep it around the level identified with $\ln V(x(t))=\zeta$ (see Fig. 10). We also observe that in the long run, the average number of packet failures approaches $\frac{2}{3}$. We remark that $\frac{2}{3}$ is a critical value for this example in the sense that $\rho<\frac{2}{3}$ in (30) implies convergence of the state, and $\sigma>\frac{2}{3}$ in (51) implies divergence.

Finally, we show that by redesigning the feedback gain $K$, we can reensure closed-loop system stability. To this end we set $K=-1.9$. It follows from Theorem 3.5 that the event-triggered control law (2), (23) with $P=1, \beta=0.01$, guarantees almost sure asymptotic stability of the closed-loop system. This can be checked as follows. By Lemma 2.3, the attack strategy (66) and its extension above satisfy (11) with $\rho_{\mathrm{M}}=0.3334$. Now, note that $p_{1}+\rho_{\mathrm{M}}<0.744$. Since $l_{\mathrm{R}}(\cdot)$ and $l_{\mathrm{M}}(\cdot)$ are not independent, it follows from Proposition 2.5 that (4) of Assumption 2.1 holds for $\rho=0.744$, which implies (26) through Lemma 3.2 Notice that Proposition 2.5 provides a tight bound $(\rho=0.744)$ for the average number of packet exchange attempt failures for the attack strategy (66) (Fig. 9). Moreover, (28)- (30) hold with $P=1, \beta=0.01$, and $\varphi=4$.

\section{CONCLUSION}

In this paper, we explored control of linear dynamical systems over networks that face random packet losses and

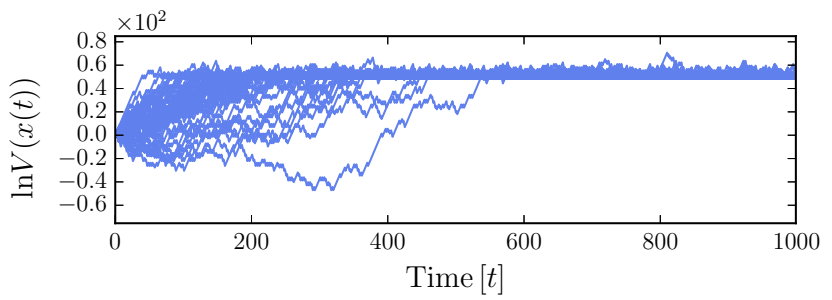

Figure 10. Sample paths of $\ln V(x(\cdot))$ under state-dependent attacks

malicious attacks. We proposed a probabilistic characterization of the evolution of the total number of packet exchange failures. Based on this characterization, we obtained sufficient conditions for almost sure asymptotic stabilization and presented a method for finding a stabilizing feedback gain and parameters for our proposed event-triggered control framework. Furthermore, to investigate potential cyber risks in networked control operations, we studied the problem from the perspective of an attacker. We obtained conditions under which combined effects of random and malicious packet losses can destabilize the closed-loop system.

The framework developed in this paper has been utilized to investigate the output feedback control problem in [54]. The probabilistic characterization developed in this paper is utilized there for modeling random and malicious packet losses in transmission of the output information from the plant sensors to the estimator in the controller side.

A direction for future research is to explore the networked control problem when wireless communication is used. There, several communication nodes and routers can be involved, and some of them may be compromised by adversaries. Our proposed network model can be incorporated to describe random failures and malicious attacks observed in such problems [55]. Furthermore, there are other important issues discussed in the networked control literature such as system and measurement noise [20], transmission delays [19], [56], and the modeling of the communication protocol [19], [45]. Investigation of these issues within our framework remains as a future work.

\section{REFERENCES}

[1] A. A. Cárdenas, S. Amin, and S. Sastry, "Research challenges for the security of control systems," in Proc. Conf. Hot Topics in Security, 2008.

[2] H. Fawzi, P. Tabuada, and S. Diggavi, "Secure estimation and control for cyber-physical systems under adversarial attacks," IEEE Trans. Autom. Control, vol. 59, no. 6, pp. 1454-1467, 2014.

[3] H. Sandberg, S. Amin, and K. H. Johansson, "Special issue on cyberphysical security in networked control systems," IEEE Control Syst. Mag., vol. 35, no. 1, 2015.

[4] W. Xu, W. Trappe, Y. Zhang, and T. Wood, "The feasibility of launching and detecting jamming attacks in wireless networks," in Proc. 6th ACM Int. Symp. Mobile Ad Hoc Network. Comput., pp. 46-57, 2005.

[5] K. Pelechrinis, M. Iliofotou, and S. V. Krishnamurty, "Denial of service attacks in wireless networks: The case of jammers," IEEE Commun. Surveys Tuts., vol. 13, no. 2, pp. 245-257, 2011.

[6] S. Amin, A. A. Cárdenas, and S. S. Sastry, "Safe and secure networked control systems under Denial-of-Service attacks," in Proc. 12th HSCC, pp. 31-45, 2009.

[7] P. Lee, A. Clark, L. Bushnell, and R. Poovendran, "Modeling and designing network defense against control channel jamming attacks: A passivity-based approach," in Control of Cyber-Physical Systems, pp. 161-175, Springer, 2013.

[8] S. Bhattacharya, A. Gupta, and T. Başar, "Jamming in mobile networks: A game-theoretic approach," J. Num. Algeb. Control Optim., vol. 3, no. 1, pp. 1-30, 2013. 
[9] H. Shisheh-Foroush and S. Martínez, "On single-input controllable linear systems under periodic DoS jamming attacks," in Proc. SIAM Conf. Contr. Appl., 2013.

[10] C. De Persis and P. Tesi, "Resilient control under denial-of-service," in Proc. 19th IFAC World Congress, pp. 134-139, 2014.

[11] C. De Persis and P. Tesi, "Input-to-state stabilizing control under denialof-service," IEEE Trans. Autom. Control, vol. 60, no. 11, pp. 2930-2944, 2015.

[12] S. Liu, P. X. Liu, and A. El Saddik, "A stochastic game approach to the security issue of networked control systems under jamming attacks," $J$. Franklin Inst., vol. 351, no. 9, pp. 4570-4583, 2014.

[13] Y. Li, L. Shi, P. Cheng, J. Chen, and D. E. Quevedo, "Jamming attacks on remote state estimation in cyber-physical systems: A game-theoretic approach," IEEE. Trans. Autom. Control, vol. 60, no. 10, pp. 2831-2836, 2015.

[14] A. T. Mizrak, S. Savage, and K. Marzullo, "Detecting malicious packet losses," IEEE Trans. Parallel Distrib. Syst., vol. 20, no. 2, pp. 191-206, 2009.

[15] T. Shu and M. Krunz, "Privacy-preserving and truthful detection of packet dropping attacks in wireless ad hoc networks," IEEE Trans. Mobile Computing, vol. 14, no. 4, pp. 813-828, 2015.

[16] A. D'Innocenzo, M. D. Di Benedetto, and F. Smarra, "Fault detection and isolation of malicious nodes in mimo multi-hop control networks," in Proc. IEEE Conf. Dec. Contr., pp. 5276-5281, 2013.

[17] S. A. Khayam and H. Radha, "Markov-based modeling of wireless local area networks," in Proc. ACM MSWiM, pp. 100-107, 2003.

[18] E. Altman, K. Avrachenkov, and C. Barakat, "A stochastic model of TCP/IP with stationary random losses," IEEE/ACM Trans. Networking, vol. 13, no. 2, 2005.

[19] L. Schenato, B. Sinopoli, M. Franceschetti, K. Poolla, and S. S. Sastry, "Foundations of control and estimation over lossy networks," Proc. IEEE, vol. 95, no. 1, pp. 163-187, 2007.

[20] J. P. Hespanha, P. Naghshtabrizi, and Y. Xu, "A survey of recent results in networked control systems," Proc. IEEE, vol. 95, no. 1, pp. 138-172, 2007.

[21] J. Xiong and J. Lam, "Stabilization of linear systems over networks with bounded packet loss," Automatica, vol. 43, pp. 80-87, 2007.

[22] H. Ishii, "Limitations in remote stabilization over unreliable channels without acknowledgements," Automatica, vol. 45, no. 10, pp. 22782285,2009 .

[23] M. Lemmon and X. S. Hu, "Almost sure stability of networked control systems under exponentially bounded bursts of dropouts," in Proc. 14th HSCC, pp. 301-310, 2011.

[24] V. Gupta, N. C. Martins, and J. S. Baras, "Optimal output feedback control using two remote sensors over erasure channels," IEEE Trans. Autom. Control, vol. 54, no. 7, pp. 1463-1476, 2009.

[25] K. Okano and H. Ishii, "Stabilization of uncertain systems with finite data rates and Markovian packet losses," IEEE Trans. Control Netw. Syst., vol. 1, no. 4, pp. 298-307, 2014

[26] A. Proano and L. Lazos, "Selective jamming attacks in wireless networks," in IEEE Int. Conf. Comm., pp. 1-6, 2010.

[27] A. Proano and L. Lazos, "Packet-hiding methods for preventing selective jamming attacks," IEEE Trans. Dependable Secure Comput., vol. 9, no. 1, pp. 101-114, 2012.

[28] B. Xiao, B. Yu, and C. Gao, "CHEMAS: identify suspect nodes in selective forwarding attacks," J. Parallel Distr. Com., vol. 67, no. 11, pp. 1218-1230, 2007.

[29] P. Tabuada, "Event-triggered real-time scheduling of stabilizing control tasks," IEEE Trans. Autom. Control, vol. 52, no. 9, pp. 1680-1685, 2007.

[30] W. P. M. H. Heemels, K. H. Johansson, and P. Tabuada, "An introduction to event-triggered and self-triggered control," in Proc. IEEE Conf. Dec. Contr., pp. 3270-3285, 2012.

[31] Q. Liu, Z. Wang, X. He, and D. Zhou, "A survey of event-based strategies on control and estimation," Syst. Sci. Contr. Eng, vol. 2, no. 1, pp. 90-97, 2014.

[32] M. Velasco, P. Martí, and E. Bini, "On Lyapunov sampling for eventdriven controllers," in Proc. IEEE Conf. Dec. Contr., pp. 6238-6243, 2009.

[33] W. P. M. H. Heemels, M. C. F. Donkers, and A. R. Teel, "Periodic event-triggered control for linear systems," IEEE Trans. Autom. Control, vol. 58, no. 4, pp. 847-861, 2013.

[34] F.-L. Qu, Z.-H. Guan, D.-X. He, and M. Chi, "Event-triggered control for networked control systems with quantization and packet losses," $J$. Franklin Inst., vol. 352, no. 3, pp. 974-986, 2015.

[35] M. Rabi and K. H. Johansson, "Scheduling packets for event-triggered control," in Proc. Eur. Contr. Conf., pp. 3779-3784, 2009.
[36] D. E. Quevedo, V. Gupta, W.-J. Ma, and S. Yuksel, "Stochastic stability of event-triggered anytime control," IEEE Trans. Autom. Control, vol. 59, no. 12, pp. 3373-3379, 2014.

[37] Y. Fang, K. A. Loparo, and X. Feng, "Stability of discrete time jump linear systems," J. Math. Systems Estim. Control, vol. 5, no. 3, pp. 275$321,1995$.

[38] J. Ezzine and D. Kavranoglyu, "On almost-sure stabilization of discretetime jump parameter systems: an LMI approach,” Int. J. Contr., vol. 68 , no. 5, pp. 1129-1146, 1997.

[39] P. Bolzern, P. Colaneri, and G. De Nicolao, "On almost sure stability of discrete-time Markov jump linear systems," in Proc. IEEE Conf. Dec. Contr, pp. 3204-3208, 2004.

[40] C. M. Kellett, I. M. Y. Mareels, and D. Nešic, "Stability results for networked control systems subject to packet dropouts," Proc. 16th IFAC World Congr., 2005.

[41] A. Cetinkaya, H. Ishii, and T. Hayakawa, "Event-triggered control over unreliable networks subject to jamming attacks," in Proc. IEEE Conf. Dec. Contr., pp. 4818-4823, 2015.

[42] L. Anantharamu, B. S. Chlebus, D. R. Kowalski, and M. A. Rokicki, "Medium access control for adversarial channels with jamming," in Proc. 18th Int. Col. SIROCCO, pp. 89-100, 2011.

[43] D. E. Quevedo and D. Nešić, "Robust stability of packetized predictive control of nonlinear systems with disturbances and Markovian packet losses," Automatica, vol. 48, no. 8, pp. 1803-1811, 2012.

[44] J. P. Hespanha and A. S. Morse, "Stability of switched systems with average dwell-time," in Proc. IEEE Conf. Dec. Contr., pp. 2655-2660, 1999.

[45] F. Smarra, A. D'Innocenzo, and M. D. Di Benedetto, "Approximation methods for optimal network coding in a multi-hop control network with packet losses," in Proc. Euro. Contr. Conf., pp. 1962-1967, 2015.

[46] A. Klenke, Probability Theory: A Comprehensive Course. SpringerVerlag, 2008.

[47] A. Karr, Probability. Springer, 1993.

[48] A. Hassibi, S. P. Boyd, and J. P. How, "Control of asynchronous dynamical systems with rate constraints on events," in Proc. IEEE Conf. Dec. Contr., pp. 1345-1351, 1999.

[49] W. Zhang, M. S. Branicky, and S. M. Phillips, "Stability of networked control systems," IEEE Contr. Syst. Mag., vol. 21, no. 1, pp. 84-99, 2001.

[50] O. L. V. Costa, M. D. Fragoso, and R. P. Marques, Discrete-Time Markov Jump Linear Systems. Springer, 2004.

[51] J. Norris, Markov Chains. Cambridge University Press, 2009.

[52] D. S. Bernstein, Matrix Mathematics: Theory, Facts, and Formulas. Princeton University Press, 2009.

[53] T. Alpcan and T. Başar, Network Security: A Decision and GameTheoretic Approach. Cambridge University Press, 2010.

[54] A. Cetinkaya, H. Ishii, and T. Hayakawa, "Event-triggered output feedback control resilient against jamming attacks and random packet losses," in Proc. IFAC NecSys, pp. 270-275, 2015.

[55] A. Cetinkaya, H. Ishii, and T. Hayakawa, "Random and malicious packet transmission failures on multi-hop channels in networked control systems," Submitted for conference publication, 2016.

[56] M. C. F. Donkers, W. P. M. H. Heemels, N. van de Wouw, and L. Hetel, "Stability analysis of networked control systems using a switched linear systems approach," IEEE Trans. Autom. Control, vol. 56 , no. 9, pp. 2101-2115, 2011.

[57] U. Madhow, Fundamentals of Digital Communication. Cambridge University Press, 2008

[58] P. Billingsley, Probability and Measure. Wiley, 2012.

\section{APPENDIX}

Lemma A.1 below provides upper bounds on the tail probabilities of sums involving a binary-valued Markov chain.

Lemma A.1: Let $\{\xi(i) \in\{0,1\}\}_{i \in \mathbb{N}_{0}}$ be a timeinhomogeneous Markov chain with transition probabilities $p_{q, r}: \mathbb{N}_{0} \rightarrow[0,1], q, r \in\{0,1\}$. Furthermore, let $\{\chi(i) \in$ $\{0,1\}\}_{i \in \mathbb{N}_{0}}$ be a binary-valued process that is independent of $\{\xi(i) \in\{0,1\}\}_{i \in \mathbb{N}_{0}}$. Assume

$$
\begin{aligned}
& p_{q, 1}(i) \leq \tilde{p}, q \in\{0,1\}, i \in \mathbb{N}_{0}, \\
& \sum_{k=1}^{\infty} \mathbb{P}\left[\sum_{i=0}^{k-1} \chi(i)>\tilde{w} k\right]<\infty
\end{aligned}
$$


where $\tilde{p} \in(0,1), \tilde{w} \in(0,1]$. We then have for $\rho \in(\tilde{p} \tilde{w}, \tilde{w})$,

$$
\mathbb{P}\left[\sum_{i=0}^{k-1} \xi(i) \chi(i)>\rho k\right] \leq \psi_{k}, \quad k \in \mathbb{N},
$$

where $\psi_{k} \triangleq \tilde{\sigma}_{k}+\phi^{-\rho k+1} \frac{((\phi-1) \tilde{p}+1)^{\tilde{w} k}-1}{(\phi-1) \tilde{p}}$ with $\phi \triangleq \frac{\rho}{\tilde{w}(1-\tilde{p})} \frac{\rho}{\tilde{p}\left(1-\frac{\rho}{\tilde{w}}\right)}$, $\tilde{\sigma}_{k} \triangleq \mathbb{P}\left[\sum_{i=0}^{k-1} \chi(i)>\tilde{w} k\right], k \in \mathbb{N}$. Moreover, $\sum_{k=1}^{\infty} \psi_{k}<\infty$.

In the proof of Lemma A.1, by following the approach used for obtaining Chernoff-type tail distribution inequalities for sums of independent random variables (see Appendix B of [57] and Section 1.9 of [58]) we use Markov's inequality. Specifically, let $y$ denote the sum of a number of random variables, and consider the tail probability $\mathbb{P}[y>\varsigma]$, where $\varsigma \in \mathbb{R}$. In obtaining a bound for this tail probability, Markov's inequality is utilized to obtain

$$
\mathbb{P}[y>\varsigma] \leq \mathbb{P}[y \geq \varsigma]=\mathbb{P}\left[\phi^{y} \geq \phi^{\varsigma}\right] \leq \phi^{-\varsigma} \mathbb{E}\left[\phi^{y}\right],
$$

for $\phi>1$. Chernoff bound is then given by $\min _{\phi>1} \phi^{-\varsigma} \mathbb{E}\left[\phi^{y}\right]$.

In the proof of Lemma A.1 we do not provide the details of the minimization process to obtain $\phi$ that gives the optimum bound. Instead, we show that the tail probability inequality (69) holds with $\psi_{k}, k \in \mathbb{N}$, and that $\sum_{k=1}^{\infty} \psi_{k}<\infty$. To obtain this result, in addition to Markov's inequality, some additional key steps (including Lemma A.2 below) are also required due to the fact that in Lemma A.1 we consider sums of (not necessarily independent) random variables composed of the product of states of a time-inhomogeneous Markov chain and a binary-valued process that satisfy (68).

Lemma A.2: Let $\{\xi(i) \in\{0,1\}\}_{i \in \mathbb{N}_{0}}$ be an $\mathcal{F}_{i}$-adapted binary-valued Markov chain with transition probability functions $p_{q, r}: \mathbb{N}_{0} \rightarrow[0,1], q, r \in\{0,1\}$. Then for all $\phi>1$, $s \in \mathbb{N}$, and $\tilde{p} \in[0,1]$ such that

$$
p_{q, 1}(i) \leq \tilde{p}, q \in\{0,1\}, i \in \mathbb{N}_{0},
$$

we have

$$
\mathbb{E}\left[\phi^{\sum_{j=1}^{s} \xi\left(i_{j}\right)}\right] \leq \phi((\phi-1) \tilde{p}+1)^{s-1},
$$

where $i_{1}, i_{2}, \ldots, i_{s} \in \mathbb{N}_{0}$ denote indices such that $0 \leq i_{1}<$ $i_{2}<\ldots<i_{s}$.

Proof: We show by induction. First, for the case $s=1$,

$$
\mathbb{E}\left[\phi^{\sum_{j=1}^{s} \xi\left(i_{j}\right)}\right]=\mathbb{E}\left[\phi^{\xi\left(i_{1}\right)}\right] \leq \phi .
$$

For the case $s=2$, the random variable $\xi\left(i_{1}\right)$ is $\mathcal{F}_{i_{2}-1^{-}}$ measurable (because $i_{1} \leq i_{2}-1$ ), and thus we have

$$
\begin{aligned}
\mathbb{E}\left[\phi^{\sum_{j=1}^{s} \xi\left(i_{j}\right)}\right] & =\mathbb{E}\left[\phi^{\xi\left(i_{1}\right)} \phi^{\xi\left(i_{2}\right)}\right] \\
& =\mathbb{E}\left[\mathbb{E}\left[\phi^{\xi\left(i_{1}\right)} \phi^{\xi\left(i_{2}\right)} \mid \mathcal{F}_{i_{2}-1}\right]\right] \\
& =\mathbb{E}\left[\phi^{\xi\left(i_{1}\right)} \mathbb{E}\left[\phi^{\xi\left(i_{2}\right)} \mid \mathcal{F}_{i_{2}-1}\right]\right] .
\end{aligned}
$$

Noting that $\{\xi(i) \in\{0,1\}\}_{i \in \mathbb{N}_{0}}$ is a Markov chain, we obtain $\mathbb{E}\left[\phi^{\xi\left(i_{2}\right)} \mid \mathcal{F}_{i_{2}-1}\right]=\mathbb{E}\left[\phi^{\xi\left(i_{2}\right)} \mid \xi\left(i_{2}-1\right)\right]$. Consequently,

$$
\begin{aligned}
& \mathbb{E} {\left[\phi^{\sum_{j=1}^{s} \xi\left(i_{j}\right)}\right]=\mathbb{E}\left[\phi^{\xi\left(i_{1}\right)} \mathbb{E}\left[\phi^{\xi\left(i_{2}\right)} \mid \xi\left(i_{2}-1\right)\right]\right] } \\
&=\mathbb{E} {\left[\phi ^ { \xi ( i _ { 1 } ) } \left(\phi \mathbb{P}\left[\xi\left(i_{2}\right)=1 \mid \xi\left(i_{2}-1\right)\right]\right.\right.} \\
&\left.\left.+\mathbb{P}\left[\xi\left(i_{2}\right)=0 \mid \xi\left(i_{2}-1\right)\right]\right)\right] \\
&=\mathbb{E} {\left[\phi ^ { \xi ( i _ { 1 } ) } \left(\phi \mathbb{P}\left[\xi\left(i_{2}\right)=1 \mid \xi\left(i_{2}-1\right)\right]\right.\right.} \\
&\left.\left.+1-\mathbb{P}\left[\xi\left(i_{2}\right)=1 \mid \xi\left(i_{2}-1\right)\right]\right)\right] \\
&=\mathbb{E}\left[\phi^{\xi\left(i_{1}\right)}\left((\phi-1) \mathbb{P}\left[\xi\left(i_{2}\right)=1 \mid \xi\left(i_{2}-1\right)\right]+1\right)\right] .
\end{aligned}
$$

Then by using (70) and (72), we arrive at

$$
\begin{aligned}
& \mathbb{E}\left[\phi^{\sum_{j=1}^{s} \xi\left(i_{j}\right)}\right] \leq \mathbb{E}\left[\phi^{\xi\left(i_{1}\right)}((\phi-1) \tilde{p}+1)\right] \\
& \quad=\mathbb{E}\left[\phi^{\xi\left(i_{1}\right)}\right]((\phi-1) \tilde{p}+1) \leq \phi((\phi-1) \tilde{p}+1) .
\end{aligned}
$$

Hence, we have that $(71)$ is satisfied for $s \in\{1,2\}$.

Now, suppose that (71) holds for $s=\tilde{s}>2$, that is,

$$
\mathbb{E}\left[\phi^{\sum_{j=1}^{\tilde{s}} \xi\left(i_{j}\right)}\right] \leq \phi((\phi-1) \tilde{p}+1)^{\tilde{s}-1} .
$$

We must show that (71) holds for $s=\tilde{s}+1$. Using arguments similar to those used for obtaining (73)-(75), we obtain

$$
\begin{aligned}
\mathbb{E}\left[\phi^{\sum_{j=1}^{\tilde{s}+1} \xi\left(i_{j}\right)}\right] & =\mathbb{E}\left[\phi^{\sum_{j=1}^{\tilde{s}} \xi\left(i_{j}\right)} \phi^{\xi\left(i_{\tilde{s}+1}\right)}\right] \\
& =\mathbb{E}\left[\mathbb{E}\left[\phi^{\sum_{j=1}^{\tilde{s}} \xi\left(i_{j}\right)} \phi^{\xi\left(i_{\tilde{s}+1}\right)} \mid \mathcal{F}_{i_{\tilde{s}+1}-1}\right]\right] \\
& =\mathbb{E}\left[\phi^{\sum_{j=1}^{\tilde{s}} \xi\left(i_{j}\right)} \mathbb{E}\left[\phi^{\xi\left(i_{\tilde{s}+1}\right)} \mid \mathcal{F}_{i_{\tilde{s}+1}-1}\right]\right] \\
& =\mathbb{E}\left[\phi^{\sum_{j=1}^{\tilde{s}} \xi\left(i_{j}\right)} \mathbb{E}\left[\phi^{\xi\left(i_{\tilde{s}+1}\right)} \mid \xi\left(i_{\tilde{s}+1}-1\right)\right]\right] \\
& \leq \mathbb{E}\left[\phi^{\sum_{j=1}^{\tilde{s}} \xi\left(i_{j}\right)}\right]((\phi-1) \tilde{p}+1) .
\end{aligned}
$$

Using (76) and (77), we arrive at (71) with $s=\tilde{s}+1$.

Proof of Lemma A.1. First, let

$$
\begin{aligned}
& \bar{\xi}(k) \triangleq[\xi(0), \xi(1), \ldots, \xi(k-1)]^{\mathrm{T}}, \\
& \bar{\chi}(k) \triangleq[\chi(0), \chi(1), \ldots, \chi(k-1)]^{\mathrm{T}}, \quad k \in \mathbb{N} .
\end{aligned}
$$

Now let

$$
F_{s, k} \triangleq\left\{\bar{\chi} \in\{0,1\}^{k}: \bar{\chi}^{\mathrm{T}} \bar{\chi}=s\right\}, s \in\{0,1, \ldots, k\}, k \in \mathbb{N} .
$$

It is clear that $F_{s_{1}, k} \cap F_{s_{2}, k}=\emptyset, s_{1} \neq s_{2}$; moreover,

$$
\mathbb{P}\left[\bar{\chi}(k) \in \cup_{s=0}^{k} F_{s, k}\right]=1, \quad k \in \mathbb{N} .
$$

It then follows that for all $\rho \in(\tilde{p} \tilde{w}, 1)$ and $k \in \mathbb{N}$,

$$
\begin{aligned}
& \mathbb{P}\left[\sum_{i=0}^{k-1} \xi(i) \chi(i)>\rho k\right]=\mathbb{P}\left[\bar{\xi}^{\mathrm{T}}(k) \bar{\chi}(k)>\rho k\right] \\
& =\sum_{s=0}^{k} \sum_{\bar{\chi} \in F_{s, k}} \mathbb{P}\left[\bar{\xi}^{\mathrm{T}}(k) \bar{\chi}(k)>\rho k \mid \bar{\chi}(k)=\bar{\chi}\right] \\
& \quad \cdot \mathbb{P}[\bar{\chi}(k)=\bar{\chi}] .
\end{aligned}
$$

Due to the mutual independence of $\xi(\cdot)$ and $\chi(\cdot)$,

$$
\mathbb{P}\left[\bar{\xi}^{\mathrm{T}}(k) \bar{\chi}(k)>\rho k \mid \bar{\chi}(k)=\bar{\chi}\right]=\mathbb{P}\left[\bar{\xi}^{\mathrm{T}}(k) \bar{\chi}>\rho k\right] .
$$


As a result, it follows from (78) and (79) that for $k \in \mathbb{N}$,

$$
\begin{aligned}
& \mathbb{P}\left[\sum_{i=0}^{k-1} \xi(i) \chi(i)>\rho k\right] \\
& =\sum_{s=0}^{k} \sum_{\bar{\chi} \in F_{s, k}} \mathbb{P}\left[\bar{\xi}^{\mathrm{T}}(k) \bar{\chi}>\rho k\right] \mathbb{P}[\bar{\chi}(k)=\bar{\chi}] \\
& =\sum_{s=0}^{\lfloor\tilde{w}\rfloor\rfloor} \sum_{\bar{\chi} \in F_{s, k}} \mathbb{P}\left[\bar{\xi}^{\mathrm{T}}(k) \bar{\chi}>\rho k\right] \mathbb{P}[\bar{\chi}(k)=\bar{\chi}] \\
& \quad+\sum_{s=\lfloor\tilde{w} k\rfloor+1}^{k} \sum_{\bar{\chi} \in F_{s, k}} \mathbb{P}\left[\bar{\xi}^{\mathrm{T}}(k) \bar{\chi}>\rho k\right] \mathbb{P}[\bar{\chi}(k)=\bar{\chi}] .
\end{aligned}
$$

In the following, we will find upper-bounds for the two summation terms in 80 . First, for the second term, since $\mathbb{P}\left[\bar{\xi}^{\mathrm{T}}(k) \bar{\chi}>\rho k\right] \leq 1, k \in \mathbb{N}$, we have

$$
\begin{aligned}
& \sum_{s=\lfloor\tilde{w} k\rfloor+1}^{k} \sum_{\bar{\chi} \in F_{s, k}} \mathbb{P}\left[\bar{\xi}^{\mathrm{T}}(k) \bar{\chi}>\rho k\right] \mathbb{P}[\bar{\chi}(k)=\bar{\chi}] \\
& \leq \sum_{s=\lfloor\tilde{w} k\rfloor+1}^{k} \sum_{\bar{\chi} \in F_{s, k}} \mathbb{P}[\bar{\chi}(k)=\bar{\chi}] \\
& =\mathbb{P}\left[\sum_{i=0}^{k-1} \chi(i)>\tilde{w} k\right]=\tilde{\sigma}_{k}, \quad k \in \mathbb{N} .
\end{aligned}
$$

Next, we look at the first term in (80). Note that $\mathbb{P}\left[\bar{\xi}^{\mathrm{T}}(k) \bar{\chi}>\right.$ $\rho k]=0$ for $\bar{\chi} \in F_{0, k}$. Hence, for all $k \in \mathbb{N}$ such that $\lfloor\tilde{w} k\rfloor=$ 0 , we have

$$
\sum_{s=0}^{\lfloor\tilde{w} k\rfloor} \sum_{\bar{\chi} \in F_{s, k}} \mathbb{P}\left[\bar{\xi}^{\mathrm{T}}(k) \bar{\chi}>\rho k\right] \mathbb{P}[\bar{\chi}(k)=\bar{\chi}]=0
$$

Furthermore, for all $k \in \mathbb{N}$ such that $\lfloor\tilde{w} k\rfloor \geq 1$, we have

$$
\begin{aligned}
& \sum_{s=0}^{\lfloor\tilde{w} k\rfloor} \sum_{\bar{\chi} \in F_{s, k}} \mathbb{P}\left[\bar{\xi}^{\mathrm{T}}(k) \bar{\chi}>\rho k\right] \mathbb{P}[\bar{\chi}(k)=\bar{\chi}] \\
& =\sum_{s=1}^{\lfloor\tilde{w} k\rfloor} \sum_{\bar{\chi} \in F_{s, k}} \mathbb{P}\left[\bar{\xi}^{\mathrm{T}}(k) \bar{\chi}>\rho k\right] \mathbb{P}[\bar{\chi}(k)=\bar{\chi}] .
\end{aligned}
$$

Now, for $s \in\{1,2, \ldots,\lfloor\tilde{w} k\rfloor\}$, let $i_{1}(\bar{\chi}), i_{2}(\bar{\chi}), \ldots, i_{s}(\bar{\chi})$ denote the indices of the nonzero entries of $\bar{\chi} \in F_{s, k}$ such that $i_{1}(\bar{\chi})<i_{2}(\bar{\chi})<\cdots<i_{s}(\bar{\chi})$. Consequently,

$$
\begin{aligned}
\mathbb{P}\left[\bar{\xi}^{\mathrm{T}}(k) \bar{\chi}>\rho k\right] & =\mathbb{P}\left[\sum_{j=1}^{s} \bar{\xi}_{i_{j}(\bar{\chi})}(k)>\rho k\right] \\
& =\mathbb{P}\left[\sum_{j=1}^{s} \xi\left(i_{j}(\bar{\chi})-1\right)>\rho k\right]
\end{aligned}
$$

for $\bar{\chi} \in F_{s, k}, s \in\{1,2, \ldots,\lfloor\tilde{w} k\rfloor\}$, and $k \in \mathbb{N}$ such that $\lfloor\tilde{w} k\rfloor \geq 1$.
Now note that $\phi>1$, since $\rho \in(\tilde{p} \tilde{w}, \tilde{w})$. We use Markov's inequality to obtain

$$
\begin{aligned}
\mathbb{P}\left[\bar{\xi}^{\mathrm{T}}(k) \bar{\chi}>\rho k\right] & \leq \mathbb{P}\left[\sum_{j=1}^{s} \xi\left(i_{j}(\bar{\chi})-1\right) \geq \rho k\right] \\
& =\mathbb{P}\left[\phi^{\sum_{j=1}^{s} \xi\left(i_{j}(\bar{\chi})-1\right)} \geq \phi^{\rho k}\right] \\
& \leq \phi^{-\rho k} \mathbb{E}\left[\phi^{\sum_{j=1}^{s} \xi\left(i_{j}(\bar{\chi})-1\right)}\right] .
\end{aligned}
$$

It follows from Lemma A.2 that $\mathbb{E}\left[\phi^{\sum_{j=1}^{s} \xi\left(i_{j}(\bar{\chi})-1\right)}\right] \leq$ $\phi((\phi-1) \tilde{p}+1)^{s-1}$. Using this inequality together with 83) and (85), for all $k \in \mathbb{N}$ such that $\lfloor\tilde{w} k\rfloor \geq 1$, we obtain

$$
\begin{aligned}
& \sum_{s=0}^{\lfloor\tilde{w} k\rfloor} \sum_{\bar{\chi} \in F_{s, k}} \mathbb{P}\left[\bar{\xi}^{\mathrm{T}}(k) \bar{\chi}>\rho k\right] \mathbb{P}[\bar{\chi}(k)=\bar{\chi}] \\
& \leq \sum_{s=1}^{\lfloor\tilde{w}\rfloor} \sum_{\bar{\chi} \in F_{s, k}} \phi^{-\rho k} \phi((\phi-1) \tilde{p}+1)^{s-1} \mathbb{P}\left[\bar{\chi}_{k}=\bar{\chi}\right] \\
& =\phi^{-\rho k+1} \sum_{s=1}^{\lfloor\tilde{w} k\rfloor}((\phi-1) \tilde{p}+1)^{s-1} \sum_{\bar{\chi} \in F_{s, k}} \mathbb{P}\left[\bar{\chi}_{k}=\bar{\chi}\right] \\
& =\phi^{-\rho k+1} \sum_{s=1}^{\lfloor\tilde{w} k\rfloor}((\phi-1) \tilde{p}+1)^{s-1} \mathbb{P}\left[\bar{\chi}_{k} \in F_{s, k}\right] \\
& \leq \phi^{-\rho k+1} \sum_{s=1}^{\lfloor\tilde{w} k\rfloor}((\phi-1) \tilde{p}+1)^{s-1},
\end{aligned}
$$

where we also used the fact that $\mathbb{P}\left[\bar{\chi}_{k} \in F_{s, k}\right] \leq 1$ to obtain the last inequality. Here, we have

$$
\begin{aligned}
\sum_{s=1}^{\lfloor\tilde{w} k\rfloor}((\phi-1) \tilde{p}+1)^{s-1} & =\frac{((\phi-1) \tilde{p}+1)^{\lfloor\tilde{w} k\rfloor}-1}{((\phi-1) \tilde{p}+1)-1} \\
& \leq \frac{((\phi-1) \tilde{p}+1)^{\tilde{w} k}-1}{(\phi-1) \tilde{p}}
\end{aligned}
$$

Hence, 86 and 87 imply

$$
\begin{aligned}
& \sum_{s=0}^{\lfloor\tilde{w} k\rfloor} \sum_{\bar{\chi} \in F_{s, k}} \mathbb{P}\left[\bar{\xi}^{\mathrm{T}}(k) \bar{\chi}>\rho k\right] \mathbb{P}[\bar{\chi}(k)=\bar{\chi}] \\
& \leq \phi^{-\rho k+1} \frac{((\phi-1) \tilde{p}+1)^{\tilde{w} k}-1}{(\phi-1) \tilde{p}},
\end{aligned}
$$

for all $k \in \mathbb{N}$ such that $\lfloor\tilde{w} k\rfloor \geq 1$. Because the right-hand side of this inequality is zero if $\lfloor\tilde{w} k\rfloor=0$, (88) holds for all $k \in \mathbb{N}$. Now, this fact together with (80), (81) leads us to 69.).

Our next goal is to show $\sum_{k=1}^{\infty} \psi_{k}<\infty$. To this end, first note that

$$
\begin{aligned}
\sum_{k=1}^{\infty} \phi^{-\rho k+1} \frac{((\phi-1) \tilde{p}+1)^{\tilde{w} k}-1}{(\phi-1) \tilde{p}} & \\
= & \frac{\phi}{(\phi-1) \tilde{p}} \sum_{k=1}^{\infty} \phi^{-\rho k}((\phi-1) \tilde{p}+1)^{\tilde{w} k} \\
& -\frac{\phi}{(\phi-1) \tilde{p}} \sum_{k=1}^{\infty} \phi^{-\rho k} .
\end{aligned}
$$


We will show that the series on the far right-hand side of 89 . are both convergent. First, since $\phi>1$, we have $\phi^{-\rho}<1$, and thus, the geometric series $\sum_{k=1}^{\infty} \phi^{-\rho k}$ converges, that is,

$$
\sum_{k=1}^{\infty} \phi^{-\rho k}<\infty
$$

Next, we show $\phi^{-\rho}((\phi-1) \tilde{p}+1)^{\tilde{w}}<1$. We obtain

$$
\phi^{-\rho}((\phi-1) \tilde{p}+1)^{\tilde{w}}=\left(\phi^{-\frac{\rho}{\tilde{w}}}((\phi-1) \tilde{p}+1)\right)^{\tilde{w}} .
$$

Furthermore,

$$
\begin{aligned}
\phi^{-\frac{\rho}{\tilde{w}}} & ((\phi-1) \tilde{p}+1) \\
& =\left(\frac{\frac{\rho}{\tilde{w}}(1-\tilde{p})}{\tilde{p}\left(1-\frac{\rho}{\tilde{w}}\right)}\right)^{-\frac{\rho}{\tilde{w}}}\left(\left(\frac{\frac{\rho}{\tilde{w}}(1-\tilde{p})}{\tilde{p}\left(1-\frac{\rho}{\tilde{w}}\right)}-1\right) \tilde{p}+1\right) \\
& =\left(\frac{\tilde{p} \tilde{w}}{\rho}\right)^{\frac{\rho}{w}}\left(\frac{1-\tilde{p}}{1-\frac{\rho}{\tilde{w}}}\right)^{-\frac{\rho}{w}}\left(\frac{1-\tilde{p}}{1-\frac{\rho}{\tilde{w}}}\right) \\
& =\left(\frac{\tilde{p} \tilde{w}}{\rho}\right)^{\frac{\rho}{\tilde{w}}}\left(\frac{1-\tilde{p}}{1-\frac{\rho}{\tilde{w}}}\right)^{1-\frac{\rho}{\tilde{w}}} .
\end{aligned}
$$

Note that $\frac{\tilde{p} \tilde{w}}{\rho}, \frac{1-\tilde{p}}{1-\frac{p}{\tilde{w}}} \in(0,1) \cup(1, \infty)$. Since $\ln v<v-1$ for any $v \in(0,1) \cup(1, \infty)$, we have

$$
\begin{aligned}
\ln & \left(\phi^{-\frac{\rho}{\tilde{w}}}((\phi-1) \tilde{p}+1)\right) \\
& =\frac{\rho}{\tilde{w}} \ln \left(\frac{\tilde{p} \tilde{w}}{\rho}\right)+\left(1-\frac{\rho}{\tilde{w}}\right) \ln \left(\frac{1-\tilde{p}}{1-\frac{\rho}{\tilde{w}}}\right) \\
& <\frac{\rho}{\tilde{w}}\left(\frac{\tilde{p} \tilde{w}}{\rho}-1\right)+\left(1-\frac{\rho}{\tilde{w}}\right)\left(\frac{1-\tilde{p}}{1-\frac{\rho}{\tilde{w}}}-1\right) \\
& =\tilde{p}-\frac{\rho}{\tilde{w}}+\frac{p}{\tilde{w}}-\tilde{p}=0,
\end{aligned}
$$

which implies that $\phi^{-\frac{\rho}{\tilde{w}}}((\phi-1) \tilde{p}+1)<1$, and hence by (91), $\phi^{-\rho}((\phi-1) \tilde{p}+1)^{\tilde{w}}<1$. Therefore,

$$
\sum_{k=1}^{\infty} \phi^{-\rho k}((\phi-1) \tilde{p}+1)^{\tilde{w} k}<\infty .
$$

Finally, (89), (90), and (92) imply $\sum_{k=1}^{\infty} \psi_{k}<\infty$. 